
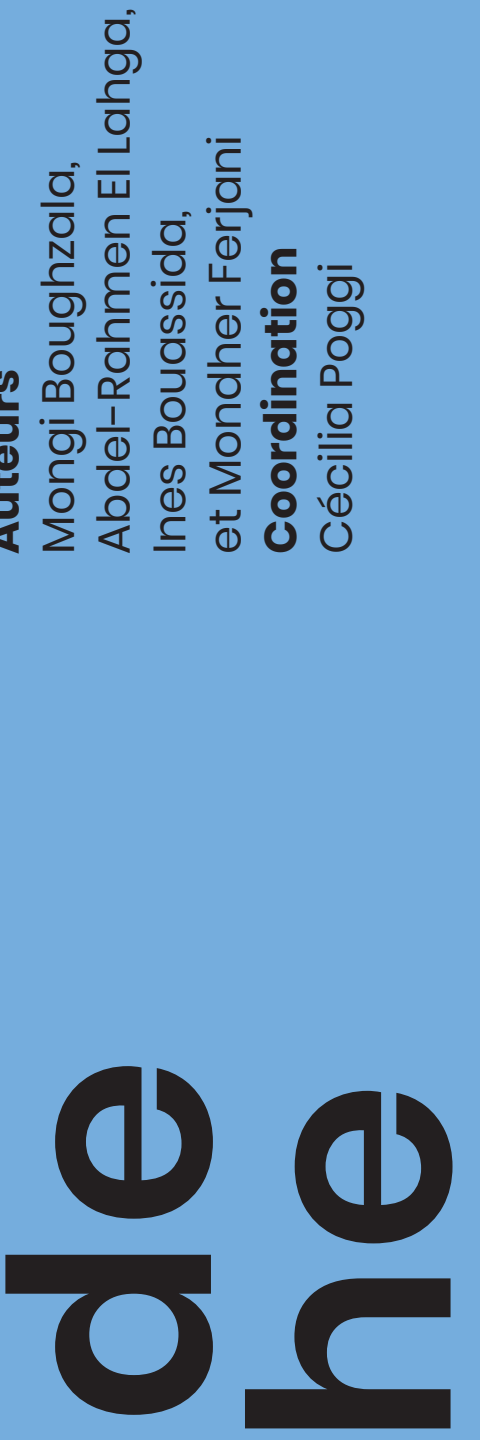

\title{
Les inégalités en Tunisie:
} quels rôles pour le capital humain hérité et le capital social?

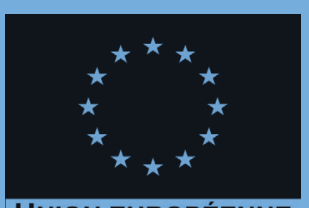





\section{Agence française de développement}

\section{Papiers de recherche}

Les Papiers de Recherche de l'AFD ont pour but de diffuser rapidement les résultats de travaux en cours. Ils s'adressent principalement aux chercheurs, aux étudiants et au monde académique. Ils couvrent l'ensemble des sujets de travail de l'AFD : analyse économique, théorie économique, analyse des politiques publiques, sciences de l'ingénieur, sociologie, géographie et anthropologie. Une publication dans les Papiers de Recherche de l'AFD n'en exclut aucune autre.

Les opinions exprimées dans ce papier sont celles de son (ses) auteur(s) et ne reflètent pas nécessairement celles de l'AFD. Ce document est publié sous l'entière responsabilité de son (ses) auteur(s)

\section{AFD Research Papers}

AFD Research Papers are intended to rapidly disseminate findings of ongoing work and mainly target researchers, students and the wider academic community. They cover the full range of AFD work, including: economic analysis, economic theory, policy analysis, engineering sciences, sociology, geography and anthropology. AFD Research Papers and other publications are not mutually exclusive.

The opinions expressed in this paper are those of the author(s) and do not necessarily reflect the position of AFD. It is therefore published under the sole responsibility of its author(s). 
Les inégalités en Tunisie:

quels rôles pour le capital humain hérité et le capital social?

Mongi Boughzala, Université de Tunis El Manar

Abdel-Rahmen El Lahga, Faculté

de Sciences Economiques

et de Gestion de Tunis, Université

de Tunis

Ines Bouassida, Faculté

de Sciences Economiques

et de Gestion de Tunis, Université

de Tunis

Mondher Ferjani, Faculté

de Sciences Economiques

et de Gestion de Tunis, Université

de Tunis

\section{Résumé}

Cet article étudie les déterminants des inégalités en Tunisie et plus particulièrement l'impact du capital humain hérité et du capital social sur les inégalités interrégionales. Le capital humain hérité d'une région ou d'une communauté englobe son savoir-faire ancestral. Cet aspect n'a pas été pris en compte jusqu'à présent pour expliquer les inégalités. Nous développons un cadre analytique permettant de clarifier la signification du capital humain hérité et du capital social et leur impact sur les revenus et les inégalités et nous le teste empiriquement à l'aide d'un modèle économétrique. Les analyses sont basées sur les données individuelles d'une enquête conduite en 2019 dans quatre gouvernorats tunisiens, ayant des niveaux de richesse différents. Nos résultats confirment l'importance du capital humain et plus particulièrement du capital humain hérité comme facteur explicatif des inégalités, notamment entre les régions. Cependant, l'incertitude n'est pas levée concernant le capital social. II en découle que la réduction des inégalités régionales nécessite une mise à niveau préalable des régions en matière de capital humain et qu'il est important d'inventorier et de valoriser le patrimoine de chaque région.

Mots-clés:

Capital humain, Capital social, Inégalités régionales, Éducation et formation

Remerciements: Cet article a été réalisé dans le cadre du Mémorandum de Dialogue Stratégique (MDS) Tunisie-AFD et de la Facilité de Recherche UE-AFD sur les Inégalités. Les auteurs expriment toute leur gratitude pourle soutien financier de I'Union européenne dont ils ont bénéficié dans ce cadre. Ils remercient les équipes de l'AFD, de l'ANPR et du CRES pour leur benveillance et leur fructueuse collaboration. Ils expriment leur appréciation de la contribution de I'Institut National de la Statistique qui a réalisé l'enquête qui a fourni les principales données utilisées dans ce travail, désormais maintenat disponibles sur demande. Leurs remerciements s'adressent aussi à tous ceux qui ont contribué à l'amélioration de ce travail par leurs remarques et critiques. Le contenu de cet article ne reflète pas nécessairement les vues de l'Union européenne, de I'AFD, du MDICI, de l'ANPR, du CRES, de I'INS et des'autres institutions partenaires.

Classification JEL : A14, B55, D31, D63, 114, 124, RII

Version originale : Français

Acceptée: Août 2020 


Abstract
This article explores the from a survey conducted in 2019 in
determinants of inequalities in four Tunisian governorates, two of
Tunisia with a focus on the impact of which lag behind when compared to
inherited human capital and social the other two. The findings confirm
capital on inequality between the significant effect of inherited
regions within the country. The human capital and of human capital
inherited human capital of a given in general. This implies that regions
community refers to its historical endowed with a weaker inherited
heritage in terms of know-how and capital need to go through an
skills, which has not been previously upgrading process as part of any
considered as a determinant of successful development program,
inequalities. The econometric model and that it is important to carry out a
is derived from a theoretical systematic inventory of each region's
framework allowing to write heritage, which may comprise
individual income as a function of valuable techniques and products
inherited human capital, social worth promoting. The impact of
capital, region dummies, and other social capital on inequality does not

seem significant and this article suggests that it should be investigated further.

Keywords:

Human capital, Social capital, Regional inequalities, Education and training 


\section{Introduction}

En Tunisie, et à travers le monde, on revendique de plus en plus un besoin de réduction des inégalités sociales et régionales. Boughzala et al., (2020) ont montré qu'en Tunisie les inégalités monétaires et non monétaires (en matière d'accès à l'éducation, la santé, l'emploi, les infrastructures) demeurent importantes (même si, jusqu'en 2015, elles pouvaient paraître relativement modérés en comparaison avec le reste du monde). Nul ne conteste en Tunisie que la réduction des disparités régionales est une priorité nationale ; le problème est de savoir comment s'attaquer au problème. Pour cela, il faut comprendre les facteurs et les mécanismes qui engendrent les inégalités et qui sont à l'origine de leur persistance. La littérature sur les causes des inégalités (voir parmi tant d'autres Gennaioli et al. 2013, Piketty 2001, Ravallion, M. 2018, IMF 2015) montre limportance de plusieurs facteurs explicatifs, notamment les ressources naturelles, la répartition initiale des richesses, le développement du capital humain, la politique fiscale et la répartition des investissements publics et privés entre les régions. Dans le présent article, l'objectif est d'étudier les déterminants des inégalités en Tunisie en portant une attention particulière aux inégalités entre les régions et de montrer que ces inégalités s'expliquent, en plus de ces dimensions, par des facteurs historiques liés au patrimoine immatériel transmis d'une génération à une autre, à savoir le capital humain hérité et le capital social.

Le capital humain hérité reflète le patrimoine immatériel, c'est-ò-dire les compétences et le savoir technologique hérités des parents, grands-parents et arrière-grands-parents. Ainsi défini, le capital humain hérité diffère d'un individu à un autre, d'une région à une autre et évidemment d'un pays à un autre. La distinction entre capital humain acquis et capital humain hérité n'a pas été prise en compte (à notre connaissance) dans la littérature, mais lidée s'inspire des travaux bien connus de Michael Porter (1990) sur l'avantage compétitif des nations, où il soutient notamment qu'au sein du même pays l'avantage compétitif des industries s'acquiert suivant un processus bien localisé dans des espaces géographiques limités et que les valeurs, la culture et l'histoire contribuent profondément à cet avantage. Notons aussi que les richesses matérielles héritées ont été prises en considération dans l'analyse des inégalités au sein d'une nation (Piketty 2001, Uslaner 2008). De même, la persistance des inégalités a été maintes fois expliquée par les mécanismes de transmission intergénérationnelle du savoir (OCDE 2018, UNICEF 2018, Onuzo et al. 2013), dans le sens que les enfants dont les parents ont fait des études avancées vont très probablement faire de même et avoir plus de chances d'accéder à de meilleurs emplois, et inversement.

Le capital social est aussi un actif historique, car il se transmet à travers les générations. Il a été largement étudié et souvent proposé comme facteur explicatif de la croissance économique (Portes 1998) et donc de la genèse et la répartition des revenus. C'est Robert Putnam, à travers ses multiples travaux (1993, 1995a, 1995b, 2000), qui a contribué le plus à populariser ce concept. Néanmoins, nos analyses s'inspirent plus des recherches de Guiso, Sapienza \& Zingales (2008a, 2010), qu'on désignera par GZS par la suite, de Tabellini (2008) et de l'OECD (2007) pour qui le capital social d'une communauté est basé sur l'ensemble des valeurs et croyances partagées par ses membres, sachant que ces valeurs et croyances déterminent le degré de cohésion, de civisme et donc de confiance mutuelle entre eux. C'est donc un élément essentiel à la réduction des risques et à l'instauration d'un climat propice à la promotion des échanges, des investissements et donc de la croissance et des revenus. L'hypothèse du capital social comme facteur explicatif des disparités régionales a été déjà mise en avant par Putnam, puis reprise et confirmée empiriquement sur la base d'une étude historique approfondie par GSZ (2008). Selon eux, l'écart entre le nord et le sud de l'Italie en matière de développement s'explique par le niveau de capital social, celui du nord étant supérieur à celui du sud.

\footnotetext{
'Les données disponibles s'arrêtent à 2015, année de l'enquête la plus récente sur le budget et la consommation des ménages.
} 
Afin d'étudier l'importance du capital humain hérité et du capital social, nous développons un cadre analytique permettant de clarifier la relation entre les inégalités, le capital humain hérité et le capital social et de définir un modèle économétrique. Ce cadre sinspire de travaux portant sur la relation entre inégalités et capital social, notamment ceux de GSZ (2008a, 2008b, 2010) et d'autres portant sur la relation entre inégalités et capital humain, avec en premier lieu le travail significatif de Gennaioli et al. (2013) qui s'appuie sur une très large base de données internationale et prend en considération à la fois le capital humain et des facteurs culturels, donc, en un sens, le capital social. Le capital humain hérité est approximé dans la définition du présent article par un index qui essaye de résumer les compétences léguées par les anciennes générations aux générations nouvelles. Cet index se base sur le niveau d'instruction du grand-père, sur son niveau de compétence et sur une mesure de la complexité de son métier. Le capital social est quant à lui interprété selon les croyances et les valeurs partagées par les membres d'une communauté et il est approximé dans la présente analyse par trois variables qui renseignent sur le niveau de coopération et de confiance perçus. L'analyse empirique est basée sur les données de l'enquête conduite en août 2019 dans quatre régions (gouvernorats) tunisiennes : celles de Monastir et de Nabeul situées à l'est dans la zone côtière relativement plus prospère et celles de Jendouba et Kasserine situées dans l'ouest moins fortuné.

Les résultats confirment limportance du capital humain hérité et de la transmission intergénérationnelle du savoir et des richesses matérielles en tant que facteurs explicatifs des inégalités, notamment entre les régions, mais ne tranchent pas concernant le rôle du capital social. Ainsi, la pauvreté relative de certaines régions s'explique en partie par leur héritage en matière de compétences et de savoir-faire et les régions côtières ont bénéficié de leur héritage (avantage) historique pour se développer plus rapidement et créer plus d'emplois. Il en découle, pour les politiques économiques, que le développement régional doit tenir compte du savoir-faire spécifique à chaque région, ville ou même localité et que la réduction des inégalités régionales devra passer en particulier par une mise à niveau systématique des régions, notamment à travers l'éducation et la formation.

Le reste de cet article est organisé en cinq parties. La première présente le contexte géographique de l'étude et donne un aperçu historique. Elle donne une description sommaire des régions (gouvernorats) couvertes par l'enquête et indique comment l'histoire aide à comprendre les disparités régionales. En partant d'une brève revue de la littérature, la seconde partie expose le cadre et le modèle théoriques et en déduit le modèle économétrique. Les données, l'échantillon du questionnaire et les principaux indicateurs sont discutés dans la troisième partie. Les résultats sont donnés en deux étapes : d'abord les résultats de l'analyse descriptive dans la partie 4 puis ceux de l'analyse économétrique dans la partie 5 . Des recommandations en matière de politique publique viennent avec la conclusion 


\section{Contexte et aperçu historique}

Cet article étudie les inégalités dans quatre régions tunisiennes, celles de Jendouba et de Kasserine à l'ouest et celles de Monastir et de Nabeul à l'est. Le nombre et le choix des régions ont été déterminés par des contraintes matérielles et par la nécessité de faire ressortir les inégalités régionales en opposant deux régions situées au bas de l'échelle de développment mesurée par l'indice de développement humain de I'ITCEQ (ITCEQ 2018) à deux régions situées au sommet de celle-ci. Cette partie donne un aperçu géographique de ces gouvernorats et s'intéresse à l'importance de l'histoire comme déterminant du présent.

Le gouvernorat de Jendouba est situé au nord-ouest ; il couvre $2 \%$ de la superficie du pays et abrite près de $3,5 \%$ de la population du pays (INS) ${ }^{2}$. Son économie est fondée sur la céréaliculture, l'élevage, les cultures maraîchères et l'arboriculture et a connu une certaine diversification depuis l'indépendance du pays (en 1956). En plus des activités agricoles qui occupent plus de la moitié de la population active, il compte 155 entreprises industrielles, principalement dans l'agroalimentaire. Le secteur du tourisme y a aussi progressé (à Tabarka et Aïndraham où on dénombre une trentaine d'unités hôtelières pour plus de 6200 lits). Ce gouvernorat est relativement bien doté sur le plan des ressources naturelles et dispose d'un important potentiel touristique et industriel.

Cependant, il est reconnu, selon les données officielles de I'INS, qu'il est parmi les plus pauvres du pays et que son niveau de développement, mesuré par l'indice de développement régional, est le plus fable du pays, au même niveau que celui de Kasserine.

Le gouvernorat de Kasserine est situé plus au sud, au centre-ouest du pays. Il occupe près de $5 \%$ de la superficie et regroupe environ $5 \%$ de la population du pays (autour d'un demimillion d'habitants). Le gouvernorat est riche en matières premières, en pierre marbrière, sable, argile et calcaire. II est aussi connu pour sa richesse archéologique : il compte 102 monuments classés par l'Institut national du patrimoine.

La majorité de la population active travaille cependant dans l'agriculture traditionnelle basée sur l'élevage et 4 \% dans l'artisanat. L'arboriculture et les cultures maraîchères, qui occupent des superficies de plus en plus importantes, se sont développées au cours des dernières décennies. Ce gouvernorat ne compte que 24 unités industrielles, dont deux entreprises à participation étrangère. II enregistre l'indice du savoir le plus faible du pays $(0,03)$, un taux de chômage très élevé, notamment parmi les jeunes et les diplômés $(35 \%$ pour les diplômés) et figure au dernier rang du classement des gouvernorats selon l'indicateur de développement régional.

\footnotetext{
2 L'Atlas des gouvernorats (MEHAT 2020) fournit les données géographiques de base sur les 24 gouvernorats du pays. Les statistiques sur la population et l'emploi sont données par l'INS.
} 
Le gouvernorat de Monastir se situe à l'est. C'est une région côtière faiblement dotée en ressources naturelles ; elle ne couvre que $0,6 \%$ de la superficie du pays et abrite plus de $5 \%$ de la population (0,6 million d'habitants). C'est l'industrie qui y emploie le plus de personnes, avec environ 1200 entreprises industrielles travaillant majoritairement dans le domaine du textile et dont la production est destinée en bonne partie à l'exportation. Ces manufactures sont implantées essentiellement aux alentours de Ksar Helal, de Bembla et de la ville de Monastir. Dans cette région, le textile représente le principal secteur d'activité depuis des siècles, surtout autour de Ksar Helal, même si l'origine historique de cette spécialisation n'est pas bien documentée.

Le secteur touristique y est important: il représente $12 \%$ de l'infrastructure touristique nationale avec 25000 lits pour 53 unités hôtelières, des parcours de golf, des terrains de tennis, des centres de plongée, un port de plaisance... Le gouvernorat bénéficie d'une infrastructure de transport relativement moderne avec un aéroport international, un métro reliant les gouvernorats de Monastir, Sousse et Mahdia.

Dans ce gouvernorat, le taux de chômage est relativement faible, nettement plus faible qu'au niveau national (moins de $10 \%$ en moyenne, au lieu de $15 \%$, et autour de $18 \%$ pour les diplômés, au lieu de plus de $30 \%$ à l'échelle nationale). Son niveau d'éducation et de développement est parmi les plus élevés du pays.

Le gouvernorat de Nabeul est situé plus au nord, dans le nord-est du pays. Il couvre moins de $2 \%$ de la superficie pays et regroupe près de $7 \%$ de la population (0,8 million habitants). Avec près de 150 hôtels et 45500 lits, soit le quart de la capacité totale d'hébergement touristique du pays, la zone de Nabeul-Hammamet se positionne comme la première zone touristique du pays.

Le développement touristique s'est accéléré après l'indépendance et s'est poursuivi avec la création du nouveau centre d'activités d'Hammamet-Sud (station balnéaire de Yasmine Hammamet), où se trouve la plus grande marina en Afrique.

L'agriculture demeure importante dans ce gouvernorat et se distingue relativement par son haut niveau d'intensification ; elle produit $15 \%$ de la production agricole nationale sur $4 \%$ de la surface agricole utile du pays, dont une forte proportion est irriguée, alors que ses ressources en eau sont insuffisantes et qu'elle dépend actuellement de l'apport provenant des eaux du nord. Les industries agroalimentaires, du bâtiment, de la céramique et des matériaux de construction y sont développées et reflètent une tradition qui remonte à plusieurs siècles. Le niveau de développement de ce gouvernorat est parmi les plus élevés du pays.

Le tableau 1 donne une idée globale de l'écart entre les gouvernorats de l'est et ceux de l'ouest. Les facteurs qui expliquent ces disparités sont multiples ; dans une certaine mesure, elles trouvent leur racine dans l'histoire. Les régions de l'intérieur du pays ont en effet connu historiquement des structures socioéconomiques différentes de celles des régions côtières et ont évolué différemment (Henia A. 2017, Habachi, M.A. 2017, Slim et al. 2006, Djait et al. 2015, 
Guellouz et al. 2015)3. Depuis l'Antiquité, les populations des régions côtières étaient plus sédentarisées et plus ouvertes au reste du monde, avec ses opportunités et ses menaces, pratiquant des activités plus diversifiées qui donnaient lieu à plus de spécialisation et d'échanges. Toutefois, la ligne de partage entre le littoral et l'intérieur est floue et très schématique. Le littoral plus fortement urbanisé se réduit dans certaines zones à une bande d'une dizaine de kilomètres le long de la côte. En outre, les régions de l'ouest du pays ont bien des villes et des villages qui ont leur histoire, et elles ont connu des périodes de prospérité et de plus forte urbanisation. Les nombreux sites archéologiques qui s'y trouvent témoignent de leur essor au cours de l'époque romaine et byzantine.

Tableau 1. Indicateurs de développement des quatre gouvernorats Sources : INS $(2014,2015,2016)$

\begin{tabular}{|l|c|c|c|c|c|}
\hline Gouvernorat & Jendouba & Kasserine & Monastir & Nabeul & Tunisie \\
\hline Taux de chômage & 25,54 & 22,35 & 9,07 & 9,95 & 14,82 \\
\hline Taux de pauvreté & 22,4 & 32,8 & 8,3 & 7,4 & 15,2 \\
\hline Taux de scolarisation 6-14 & 93,92 & 90,07 & 96,43 & 96,73 & 97,62 \\
\hline Taux de scolarisation 19-24 & 38,95 & 32,35 & 97,62 & 36,26 & 40,87 \\
\hline $\begin{array}{l}\text { Niveau de dépense moyen (en 1 000 } \\
\text { dinars) }\end{array}$ & 2943 & 2543 & 5115 & 3919 & 3871 \\
\hline Indice de développement humain & 0,23 & 0,24 & 0,51 & 0,73 & \\
\hline
\end{tabular}

Jusqu'à la veille de l'indépendance (1956), une bonne partie de la population rurale du centre-ouest du pays alternait entre vie sédentaire et vie nomade et pratiquait régulièrement la transhumance vers le nord-ouest où les terres sont plus fertiles et les récoltes plus régulières, car l'élevage constituait sa principale activité. Jusqu'à la fin du XIXème siècle, les rapports des populations (tribus) de l'ouest avec les autorités centrales étaient plutôt tendus et se réduisaient souvent au versement de l'impôt, à l'allégeance des chefs de tribu aux monarques de l'époque et éventuellement au recrutement de guerriers parmi leurs jeunes. Les autorités beylicales (début du XVIIIème à la fin du XIXème siècles), et d'autres dynasties avant elles, n'avaient pas œuvré pour unir ces tribus et les intégrer ; elles avaient plutôt cherché à les diviser et à renforcer les hostilités entre elles en vue de les affaiblir et de les dominer plus facilement. La colonisation française (1881-1956) les a appauvries encore plus en les dépossédant d'une partie de leurs terres. Un exode massif vers les faubourgs des villes situées pour la plupart à l'est du pays (Tunis en premier lieu) en a résulté et s'est poursuivi après l'indépendance à cause du développement inégal entre les régions.

\footnotetext{
${ }^{3}$ Cet aperçu historique est évidemment très sommaire. Une étude plus approfondie de l'histoire des régions du nord et du centreouest comparée à celle des régions côtières mérite d'être entreprise, et ce à l'instar de l'étude de Guiso et al. (2008b) portant sur l'histoire médiévale de l'Italie et la comparaison entre le sud et le nord de l'Italie en matière de développement et de niveau de capital social.
} 
De nos jours, toutes les régions du pays sont régies par les mêmes lois et le même type d'institutions locales et régionales, mais leur passé laisse son empreinte sur le présent. Quoique leurs activités se sont diversifiées, elles ont évolué d'une manière très inégale, avec une forte concentration à l'est des investissements privés créateurs d'emplois et de richesse.

On peut songer à expliquer historiquement les différences en matière de développement par l'évolution de leurs autorités locales et régionales, et ce en s'inspirant de la conjecture de Putnam reprise et testée par Guiso et al. (2008b). Selon cette conjecture, l'avantage du centre et du nord de l'Italie résulte des circonstances historiques qui ont prévalu du MoyenÂge jusqu'à l'unification de l'Italie à la fin du XIXème siècle et y ont favorisé l'émergence de communes libres et prospères et concomitamment le développement du capital social. En effet, le centre et le nord avaient l'avantage de l'autonomie contrairement au sud qui était unifié et soumis au pouvoir central exercé par la dynastie des Normands.

Cette piste ne semble pas assez prometteuse dans le contexte tunisien. Les régions de l'ouest tunisien étaient en fait plus décentralisées que celles de l'est et moins soumises à l'autorité centrale. Cependant, les conditions n'y étaient pas propices au développement et à la création d'entités locales puissantes et efficaces. Mis à part dans les villes, ces régions étaient organisées en tribus trop divisées. En revanche, l'État central, quoique plus présent dans les régions de l'est, n'y était guère prédominant. Certaines communautés de l'est ont réussi à préserver leur patrimoine et à organiser leurs activités dans le cadre non pas d'autorités politiques locales mais d'institutions spécifiques : des corporations de métiers, des marchés et circuits de distribution pour leurs produits comme les tissus ou la poterie, ce qui suppose un niveau de capital social adéquat.

La mise en place systématique d'autorités municipales modernes n'a été généralisée qu'après l'indépendance, mais tout en les soumettant jusqu'à 2010 à une lourde tutelle du pouvoir central et à la mainmise du parti unique. La nécessité de la décentralisation et d'une approche participative du développement est finalement reconnue et consacrée par la nouvelle constitution de 2014, mais tout reste à faire en pratique pour instaurer de véritables autorités décentralisées susceptibles d'œuvrer pour le développement et la réduction des inégalités régionales. 


\section{Revue de la littérature et cadre théorique}

\subsection{Revue de la littérature}

Les inégalités sociales ont toujours existé, entre les pays et au sein de tous les pays, et il y a toujours eu partout une certaine concentration des richesses et des moyens de production dans les mains d'une minorité, mais pas toujours au même niveau. Dans les pays d'Europe occidentale, notamment en France (Piketty 2001 et 2013), le niveau des inégalités a nettement baissé au cours du XXème siècle. Plusieurs facteurs sont avancés pour expliquer le niveau et l'évolution des inégalités. Piketty (2013) souligne l'effet de la progressivité de l'impôt sur les revenus. Bourguignon (2016) et Milanovic (2016) montrent séparément que la baisse des inégalités intra-pays observée entre 1820 et 1990 n'est pas spécifique aux pays d'Europe occidentale, que les inégalités à l'échelle mondiale, entre pays, ont augmenté au cours de cette même période et que, depuis la fin du XXème siècle, cette tendance s'est inversée : moins d'inégalités à l'échelle mondiale et plus d'inégalités à l'intérieur des pays, avec des exceptions notables. Bourguignon (2016), Milanovic (2016), Ravallion (2018) et Acemoglu (2003) ont étudié le rôle de la libéralisation commerciale et de la transition technologique pour expliquer cette évolution.

Des études micro-économétriques ont permis d'identifier d'autres facteurs explicatifs des inégalités entre les régions. Gennaioli et al. (2013) ont analysé l'effet du capital humain et des facteurs géographiques, culturels et institutionnels. Ils ont montré que le facteur géographique lié à l'emplacement de la région et des ressources naturelles a un effet significatif sur les inégalités, mais que le principal facteur explicatif réside dans le capital humain et les externalités qui lui sont associées. En revanche, la confiance (indissociable du capital social) et l'hétérogénéité ethnique sont assez significatives entre pays, mais le sont peu au niveau régional à l'intérieur d'un même pays.

Le rôle déterminant du capital humain ne fait que confirmer ce qui a été démontré et souligné de plusieurs manières par tant d'autres contributions notoires depuis Becker (1964) et Shultz (1961), dont celles de Barro (1991), Mankiw, Romer et Weil (1992), Krueger et Lindahl (2001), De la Fuenta et Domenech (2006), Acemoglu et Dell (2010). De nombreux travaux ont montré que le niveau d'éducation des parents et le contexte dans lequel un individu est élevé déterminent ses chances sur le marché du travail et donc son revenu (OCDE 2018, UNICEF 2018, Onuzo et al. 2013).

Quant au rôle du capital social, quoiqu'il soit assez souvent étudié, il n'a pas été analysé dans le contexte des inégalités en Tunisie. La première définition donnée du capital social est axée sur l'appartenance à des réseaux permettant à leurs membres de se connaître et de bénéficier mutuellement de divers avantages (Bourdieu, 1985). Cette idée est élargie à l'appartenance à une communauté (Coleman, 1990). Le capital social a été aussi défini comme l'ensemble des relations qui maintiennent les normes sociales bénéfiques. Selon Putnam (1993 et 1995), il se manifeste essentiellement par le respect de l'intérêt commun et de la loi et par l'honnêteté et le dévouement des responsables de l'intérêt public. La définition retenue dans le présent article est proposée par Guiso et al. (2013). Elle se détache de 
l'appartenance à des réseaux ou à des clubs et souligne qu'il s'agit d'un bien public et non d'un bien privé et ramène le capital social d'une communauté au stock de valeurs et croyances durables (ou civisme) qui favorisent l'intérêt commun et la coopération entre les membres de cette communauté. Ces valeurs constituent un bien public et sont transmises d'une génération à une autre. Elles risquent ainsi de se déprécier, car la transmission n'est généralement pas parfaite ; l'accumulation de ce capital est possible à travers l'investissement, notamment dans le système éducatif.

Sur le plan empirique, il existe de plus en plus de travaux qui mesurent et utilisent le capital social dans des contextes très divers, mais leur nombre reste modeste. On peut citer Gennaioli et al. (2013) qui étudient le lien entre inégalités et capital social. Plusieurs études se concentrent sur la relation croissante entre le capital social et l'accès aux principaux services sociaux, tels que l'éducation, la santé et l'emploi (voir par exemple Cook 2014 et Dufur et al. 2013).

On retiendra de cette brève revue de la littérature que la répartition initiale des richesses, le développement du capital humain, la qualité des services publics et de l'infrastructure de base, l'accès au système financier, la politique fiscale et de redistribution sont des facteurs explicatifs des inégalités. Boughzala et al. 2020 ont souligné l'importance de ces facteurs dans le cas tunisien. Le but du présent article n'est pas de revenir sur ces divers facteurs, mais de s'intéresser au rôle du capital humain hérité et du capital social sur la base d'un cadre théorique qui s'inspire de plusieurs travaux existants.

\subsection{Cadre théorique}

Le cadre théorique s'inspire mais ne reprend intégralement aucun des modèles déjà proposés. II s'appuie en premier lieu sur les travaux de Guiso et al. (2010 et 2008a, 2008b) concernant le capital social et de Gennaioli et al. (2013) pour ce qui est des déterminants des inégalités régionales. Ces derniers cherchent à étudier les déterminants des inégalités spatiales et ont l'avantage de disposer d'une base de données plus riche que la nôtre. Ils sont partis d'un modèle d'équilibre général décrivant le comportement et les choix des entreprises et des ménages et donnant lieu à une allocation en matière d'emplois, d'investissement, de production, de revenus et de localisation. Notre modèle adopte aussi une approche d'équilibre général, mais prend une forme bien plus réduite adaptée aux données disponibles et au contexte tunisien. II s'inspire des travaux de GSZ pour ce qui est du capital humain et de sa dynamique.

Le point de départ consiste à définir les principaux acteurs, le cadre spatial et le concept de communauté. Partant du fait que la Tunisie est composée de régions sous forme de gouvernorats, tout individu interrogé (le chef de ménage) est appelé à déclarer la communauté à laquelle il appartient - il peut appartenir à un groupe qui peut se réduire à un noyau familial ou s'étendre à un village ou à un quartier, à une ville, à sa région ou enfin à tout un pays. En général, il ne va effectivement agir et interagir avec d'autres individus que dans une partie du pays. Cela dépend de sa mobilité, de la nature de ses activités et de ses liens sociaux. Nous appelons communauté l'ensemble des individus appartenant à un 
espace bien défini qui interagissent entre eux. La communauté peut être plus ou moins étendue ; sa taille indique les possibilités d'action des individus qui lui appartiennent.

De quelles interactions s'agit-il ? Tout individu a besoin de partenaires, notamment pour l'échange de biens et de services, la réalisation de projets d'investissement, l'emploi, l'emprunt ou la vente à crédit ou à terme. Nous nous intéressons ici principalement aux transactions liées à la création de revenus, donc au comportement des individus en tant qu'investisseurs et producteurs, employeurs de capital physique et humain, et en tant que travailleurs et fournisseurs de capital humain. C'est de cela que dépendent les revenus et les inégalités sociales et régionales (ou spatiales).

Dans ce contexte, la confiance entre les individus est importante, car elle réduit les frais d'assurance et les coûts de transaction, ainsi que les pertes subies en cas de partenaires incompétents ou malhonnêtes. La confiance est indissociable de l'honnêteté et donc du capital social.

La réalisation et le succès des activités productives dépendent de deux types de probabilités.

i) D'abord, de la chance (la probabilité PI) de trouver les partenaires susceptibles d'offrir ce qu'on cherche.

ii) Ensuite, la probabilité (P2) que ces partenaires soient en effet dignes de confiance.

Le premier type de condition concerne la probabilité de trouver des partenaires ayant le profil recherché, notamment des employés ou des fournisseurs ayant le capital humain ou le savoir-faire recherché, ce qui dépend de l'offre de capital humain.

Le deuxième type de condition concerne les valeurs intrinsèques des partenaires trouvés et du degré de confiance qu'on peut leur accorder. Vont-ils agir honnêtement et coopérer au cours de toutes les étapes des transactions envisagées ? II s'agit donc de la probabilité P2 qu'ils soient dignes de confiance.

Au temps $t$, la communauté comprend $\mathrm{n}$ individus actifs, dont $\mathrm{m}$ entrepreneurs ( $\mathrm{m}$ chefs d'entreprise/entreprises employant des salariés, dont le nombre peut être petit, moyen ou grand). Le nombre $m$ est donné au temps $t$, mais pourra évoluer ensuite : des entreprises peuvent se délocaliser vers ou en dehors de la région ou disparaître, d'autres peuvent être créées. De même, pour le nombre n, il pourra changer à la suite de mouvements migratoires interrégionaux.

$$
\Delta n=f(E(w-\bar{w}))
$$

Le flux migratoire se fait conformément à l'hypothèse de Harris-Todaro (1970), c'est-à-dire que la variation de $\mathrm{n}$ est une fonction croissante de l'écart entre le bien-être espéré $\mathrm{E}(\mathrm{w})$ dans la région (ou communauté) et le bien-être espéré moyen à l'échelle nationale $\mathrm{E}(\bar{w})$. Si cet écart est positif, $n$ augmentera (il y aura un flux migratoire vers la région) et inversement. 
Le bien-être tient compte non seulement du revenu, mais aussi des conditions de logement et d'accès aux divers services publics ${ }^{4}$.

Concernant la détermination du nombre d'entreprises $m$, Gennaioli et al. (2013) supposent dans leur modèle que les individus procèdent à un choix optimisé entre un emploi salarié et un emploi de manager/entrepreneur et que les mêmes compétences peuvent servir à occuper un emploi managérial ou un emploi salarié ordinaire. Nous considérons que pour être un entrepreneur, il faut avoir des aptitudes moins communes, acquises surtout à travers l'héritage familial matériel et immatériel. En effet, à partir des données que nous avons collectées (et qui seront présentées dans la partie suivante), nous trouvons que seul le capital humain hérité (khh), que nous allons définir plus loin, explique la probabilité d'être chef d'entreprise et que même le niveau d'éducation de l'individu ne semble pas significatif, quoique cela n'implique pas que des études avancées ne soient pas un pré-requis pour des postes de management dans les grandes entreprises modernes (voir le tableau A 1 pour la régression logistique de l'entrepreneuriat en fonction du capital humain hérité et acquis).

En outre, en Tunisie, le choix du type d'éducation (et donc de capital humain) est à peine le résultat d'un choix optimal effectué par l'individu; c'est surtout le produit du système éducatif qui laisse peu de liberté aux individus.

Le niveau et la répartition des revenus va découler du comportement des individus en tant que chefs d'entreprise et/ou chefs de ménage.

\section{- Comportement des entreprises}

Les entreprises, agissant dans un environnement risqué, vont maximiser une fonction de gain qui dépend positivement du profit qu'elles peuvent réaliser sur un certain horizon et négativement du risque associé à la probabilité que leurs partenaires ne soient pas dignes de confiance (ils risquent de tricher et/ou de ne pas fournir l'effort attendu d'eux indépendamment des incitations qui leur sont offertes). Autrement dit, leur fonction de gain est une fonction croissante du profit espéré et du capital social caractérisant leur communauté, car plus de capital signifie moins de risque. Soit $k s_{j}$ le niveau de ce capital social tel qu'il est perçu par l'entreprise $j$. On reviendra à sa définition et à sa mesure dans cette partie.

La solution du problème de l'entreprise $j$, conditionnée par la valeur de $k s_{j}$, donne la valeur des variables suivantes : $Y_{j}$ qui désigne son niveau de production, $L_{j}$ le nombre de travailleurs qu'elle va employer, $K H_{j}$ et $K_{j}$, respectivement, le volume de capital humain et de capital physique qu'elle va employer. Le capital humain couvre le savoir, les aptitudes, les compétences et toutes les valeurs qui permettent à un travailleur de produire plus et mieux. L'accumulation de $K_{j}$ au cours de la période actuelle donne l'investissement de l'entreprise. Cette accumulation (investissement) peut être négative si l'entreprise décide de se délocaliser partiellement ou totalement. Pour les mêmes raisons, $L_{j}$ peut augmenter ou

${ }^{4}$ Gennaioli et al. modélisent les flux migratoires autrement en combinant le revenu et le coût du logement. 
diminuer. La localisation et la délocalisation pour une entreprise sont déterminées en fonction du gain espéré et de la taille de la communauté où elle opère.

Dans ce qui suit, $k$ et $k h$ désignent, respectivement, le capital physique et le capital humain par employé ; $k=K / L, k h=K H / L$ et $y=Y / L$ désignent la productivité moyenne.

On peut écrire :

$$
y_{j}=f\left(k h_{j}, k_{j} ; k s_{j}, z_{j}\right), z_{j} \text { indique les autres déterminants de } y_{j} .
$$

En supposant qu'il y a une forte complémentarité entre $k h_{j}$ et $k_{j}$, on peut écrire:

$$
y_{j}=y j\left(k_{j} ; k s_{j}, z_{j}\right)
$$

Le revenu obtenu par le chef d'une entreprise et celui obtenu par ses employés sont déterminés par la productivité de cette entreprise.

Le revenu de l'individu $i,\left[R_{i}(i=1, \ldots, n)\right]$ dépend, directement et indirectement, de la productivité de l'entreprise où il travaille comme chef d'entreprise ou comme employé, ainsi que du capital humain qu'il lui fournit ( $h i$ ). Qu'ils soient des salariés ou des entrepreneurs, les revenus obtenus par les individus sont donc, d'une manière ou d'une autre, liés à $k$, hi et $k s^{5} 6$.

$$
R_{i}=r\left(k_{i}, k s_{i}, h i, z_{i}\right)
$$

$i$ renvoie à l'entreprise où l'individu $i$ travaille; $h i$ est le capital humain fourni par l'individu i à l'entreprise où il travaille. On suppose que le capital social tel qu'il est perçu au niveau de l'entreprise où i travaille $\left(k s_{i}\right)$ est le même que celui perçu par l'individu $i$.

\section{- Comportement des individus en tant que chefs de ménage}

Les individus (à l'âge actif) sont caractérisés par leur niveau d'éducation, ou capital humain acquis ( $k h a$ ), les actifs physiques qu'ils ont accumulés ou hérités (du capital physique et des terres) et un savoir-faire ancestral hérité que nous appelons capital humain hérité ( $k h h)$. II s'agit des compétences et du savoir-faire reçus dans le contexte familial qui reflètent les compétences ancestrales propres à leur famille et/ou à leur communauté et transmis d'une génération à une autre. Le capital humain d'un individu est donc composé de son capital humain acquis et de son capital humain hérité.

Le problème des individus, en tant que chefs de ménage, est de décider comment exploiter leurs actifs, où travailler, quel effort attribuer à l'éducation de leurs enfants et éventuellement s'il faut rester dans la même région ou émigrer. Leur revenu provient de leur travail dans une entreprise en tant qu'employé ou chef d'entreprise. En général, les chefs

\footnotetext{
${ }^{5}$ Sans avoir besoin de supposer que les marchés convergent vers un équilibre et vers l'égalisation des productivités marginales des facteurs à leur prix réel.

${ }^{6}$ Par ailleurs, l'augmentation de la productivité moyenne des entreprises de la communauté peut avoir un effet externe positif sur tous les revenus et sur les ressources publiques et donc sur la disponibilité et la qualité des services publics.
} 
d'entreprise possèdent et/ou ont hérité d'un patrimoine important en matière de capital physique et/ou humain.

Les individus vont transmettre à leurs enfants, plus ou moins intégralement, leur patrimoine matériel et immatériel et investir dans leur éducation, de la même manière qu'ils ont reçu de leurs parents une partie de leur patrimoine matériel et immatériel et bénéficié de ce que leurs parents ont consenti pour leur éducation. Cependant, l'éducation formelle reçue par les enfants dans les écoles dépend plus de la possibilité d'accès au système éducatif et de sa qualité que de l'effort fourni par les parents.

Ainsi, le capital humain dont dispose un individu résulte de son capital humain acquis $\boldsymbol{k} \boldsymbol{h a}$ et de son capital humain hérité $\boldsymbol{k} \boldsymbol{h} \boldsymbol{h}$. Le capital humain offert par l'individu $\boldsymbol{i}\left(h_{i}\right)$ peut être écrit sous la forme:

$$
h_{i}=h\left(k h a_{i}, k h h_{i}\right)
$$

Nous supposons que l'équilibre atteint sur le marché du travail n'est pas nécessairement le plein emploi (l'objectif de cet article n'est pas d'étudier le fonctionnement du marché du travail) et que le revenu de chaque individu provient de la rémunération de son travail et de son capital humain.

En supposant que chaque individu offre une unité de travail, on peut réécrire l'équation (3), l'équation du revenu, sous une forme qui synthétise le comportement de l'entreprise et le comportement de l'individu chef de ménage:

$$
R_{i}=r\left(k_{i}, k h a_{i}, k h h_{i} ; k s_{i}, z_{i}\right)
$$

Il est à noter que l'étude de $R_{i}$ à travers l'estimation de cette équation permet d'examiner les inégalités et leurs déterminants non seulement entre individus, mais aussi entre groupes d'individus (par exemple groupés par région, décile, niveau d'éducation ou genre). Elle permet d'analyser la distribution $\left(R_{i}\right)$ en fonction des variables $k_{i}, k h a_{i}, k h h_{i} ; k s_{i}, z_{i}$.

Dans la partie empirique, $z_{i}$ sera une indicatrice de la région de l'individu $i$.

\section{- Mesure et dynamique du capital social}

Le capital social d'une communauté est défini comme l'ensemble des valeurs et des croyances qui favorisent la coopération et la cohésion entre ses membres. Il se manifeste essentiellement par le degré de civisme et d'honnêteté (ne pas tricher, ne pas voler, ne pas mentir, être fidèle à ses engagements et respecter le bien et l'espace communs) qui caractérise l'ensemble de la communauté et détermine le niveau de confiance qui règne entre ses membres. En particulier, le capital social facilite les relations entre les partenaires d'une entreprise (employés, fournisseurs, clients, créanciers, managers). Plus le capital social est grand, plus les gens se font confiance et coopèrent facilement entre eux.

Si tous les membres de la communauté étaient parfaitement honnêtes la confiance régnerait partout et tout le monde serait rassuré et croirait que tous les autres sont dignes de confiance?... En réalité, il n'y a pas de communauté parfaitement homogène et honnête.

${ }^{7}$ II existe évidemment d'autres formes et sources de risques liés à d'autres aléas qui dépassent le cadre de cette étude. 
Certains individus risquent de tricher et de ne pas respecter leurs engagements et la probabilité qu'un individu tiré au hasard respecte ses engagements suit une distribution qui varie d'une communauté à une autre. Cette distribution permet de calculer le niveau de confiance moyen (espéré) de la communauté $(P)$ qui est adopté comme indicateur du capital social.

Pour calculer $P$, on suppose que les $n$ individus sont répartis en 6 groupes, comprenant chacun $n_{s}$ individus. Le groupe 0 est composé des $n_{0}$ individus auxquels on attribue la valeur 0 parce qu'ils refusent de s'engager auprès d'autres à moins que toutes les garanties soient assurées. Ce sont les individus qui ne s'engagent pas, non parce qu'ils ne sont pas dignes de confiance, mais parce qu'ils ont été tellement découragés et déçus qu'ils deviennent extrêmement méfiants. Les cinq autres groupes vont du groupe 1 composé de $n_{1}$ individus qui ne respectent pas leurs engagements et auxquels on attribue la valeur 1 au groupe 5 composé de $n_{5}$ individus qui sont tout à fait honnêtes et qui respectent parfaitement leurs engagements et auxquels on attribue la valeur 5. $n=\sum_{s} n_{s}$

Il y a donc une probabilité $P_{s}=n_{s} / n$ de rencontrer au hasard un individu du groupe $s, s=$ $0,1,2 \ldots .5$.

D’où le niveau de confiance espéré $(P)$,

$$
P=\sum_{s} s n_{s} / n \text { et } 1 \leq P \leq 5
$$

$P$ dépend de la structure des valeurs, reflétée par $S=\left(s_{0}, s_{1}, s_{2}, \ldots, s_{5}\right)$, et de la structure sociale de la communauté, indiquée par $N=\left(\boldsymbol{n}_{0}, \boldsymbol{n}_{1}, \boldsymbol{n}_{2}, \ldots, \boldsymbol{n}_{5}\right)$.

Notons que $n$ et les $n_{s}$ peuvent varier dans le temps ; $n$ varie selon le mécanisme décrit par l'équation (1), $\Delta n=f(E(w-\bar{w}))$; quant aux $n_{s}$, ils varient parce que la structure de la société peut changer, notamment sous l'effet de l'éducation, de la migration et d'autres changements démographiques. Les mouvements migratoires de groupes qui ne partagent pas nécessairement les mêmes valeurs peuvent modifier le niveau du capital social.

Les travaux existants tendent à supposer que la communauté ou la société est homogène et composée d'individus semblables qui optimisent leur choix concernant le niveau du capital social. Nous voyons que le capital social est un bien public qui fait l'objet d'un choix public. GSZ supposent que les ménages décident du niveau optimal de confiance (donc du capital social) à apprendre à leurs enfants. Nous considérons que le niveau de confiance change essentiellement sous l'effet du changement de la structure sociale, alors que les valeurs fondamentales sont plutôt stables. Les valeurs, comme les notions de bien et de mal, constituent un code de comportement social et donc un bien public qui résulte d'un choix collectif et non de choix individuels; elles forment un socle social forgé par un long processus historique. GSZ (2008b) eux-mêmes, ainsi que Uslaner (2008), soutiennent que les valeurs bénéfiques ont persisté et façonné le capital social pendant des siècles, respectivement en Italie du centre et du nord et aux États-Unis. Nous voyons que les parents transmettent à leurs enfants non un niveau de confiance, mais des valeurs qu'ils ont euxmêmes reçues de leurs parents à travers leur éducation. Les parents s'adaptent au cours de leur vie à l'évolution du niveau de confiance due au changement des structures sociales en modifiant leur comportement et non leurs valeurs. 
Toutefois, les valeurs ne sont guère transmises par les parents aux enfants de manière mécanique et parfaite. De ce fait, le capital social risque de se déprécier et de se dégrader si rien n'est fait collectivement à travers le système éducatif pour compenser cette dépréciation.

En somme, la valeur de $P$ évolue dans le temps selon la dynamique adaptative suivante:

$$
\begin{gathered}
P_{t}=P_{t-1}(1-\delta)+G\left(\Delta N_{t}\right)+g\left(I s, \varepsilon_{t}\right) \\
\Delta P_{t}=G\left(\Delta N_{t}\right)+g\left(I s, \varepsilon_{t}\right)-\delta P_{t-1}
\end{gathered}
$$

$\delta$ indique le taux de dépréciation du capital social, $\Delta N_{t}$ la variation de la structure sociale provoquée par des mouvements démographiques et migratoires et Is le niveau des investissements publics destinés à l'amélioration du capital social, notamment à travers l'éducation et le maintien de l'État de droit. L'équation (8) décompose la variation de $P$ en trois composantes, la première reflète ce qui est dû à la modification de la structure sociale, la seconde à l'effet de l'investissement public, la troisième à la dépréciation naturelle. Le niveau de $P_{t}$ peut baisser sous l'effet de la dépréciation naturelle $(\delta)$, de la détérioration de la qualité du système éducatif et/ou de l'État de droit (Is) ou du changement de la structure de la population $\left(\Delta N_{t}\right)$, y compris à cause de la migration si ceux qui migrent ne partagent pas les mêmes valeurs. Notons que la durée de temps entre $t$ et $(t-1)$ n'est normalement pas l'année, car les valeurs et la structure sociale évoluent lentement.

Tabellini (2013) s'est plus particulièrement intéressé à la dynamique du capital social, mais selon une approche et un cadre différents des nôtres. II a montré la possibilité de sa convergence vers un équilibre stable, mais qui risque, si on part d'un niveau de confiance et de capital social faible, d'être un équilibre défavorable, où le capital social s'affaiblit progressivement et où la suspicion est de plus en plus fréquemment la règle, donc vers un équilibre où la méfiance génère plus de méfiance et où l'investissement et les échanges sont de plus en plus difficiles. Notre modèle ne permet pas de reproduire le même résultat. Le capital social peut se détériorer sans converger vers un équilibre défavorable et inversement.

Empiriquement, il ne nous est pas possible d'estimer ou de bien calibrer cette équation. Les éléments d'information disponibles permettent néanmoins de l'interpréter et d'en tirer une proposition qui pourrait faire ultérieurement l'objet d'investigations plus approfondies. L'analyse empirique sera pour l'essentiel en deux parties, l'une descriptive et l'autre économétrique, basée principalement sur l'estimation de l'équation 5. 


\section{Les données}

\section{1. Échantillon et questionnaire}

Les données nécessaires proviennent d'une enquête conduite en Tunisie entre juillet et août 2019. L'objectif principal de l'enquête réalisée pour la préparation de cet article était de recueillir des données indispensables pour mesurer le capital hérité et le capital social et estimer leur impact sur les inégalités. Cette enquête, la première de son genre en Tunisie, a été financée par la Facilité de Recherche UE-AFD sur les Inégalités, avec le soutien financier de I'Union européenne, et exécutée par l'Institut National de Statistique (INS), en collaboration avec les auteurs de cet article.

Le territoire tunisien est découpé en districts géographiques de recensement qui regroupent chacun un ensemble d'unités primaires d'échantillonnage (UPE). Dans chaque délégation choisie pour notre enquête, 50 UPE ont été tirées au hasard. Dans une seconde étape, 10 ménages de chaque UPE sont tirés au hasard. Ainsi, pour chaque région, nous avons 500 ménages visités. Sur l'ensemble des 2000 ménages ciblés par l'enquête, 1963 questionnaires ont été renseignés totalement ou partiellement. L'échantillon comprend des ménages répartis entre des communautés (délégations) appartenant aux quatre gouvernorats de Jendouba, Kasserine, Monastir et Nabeul (voir tableau 2).

Le chef de ménage et son conjoint (ou sa conjointe) sont interrogés (face à face) pour fournir des informations les concernant directement et concernant leurs parents, grandsparents et enfants, y compris ceux qui ont quitté le foyer parental. Ces informations portent surtout sur le niveau d'éducation et de compétence, la profession, les activités, le capital social et les revenus répartis par source telle que le travail, les propriétés. Le questionnaire élaboré à cet effet s'articule autour de 6 modules.

Tableau 2. Nombre de ménages par gouvernorat

Source : calculs des auteurs à partir de l'enquête sur les inégalités en Tunisie, INS 2019

\begin{tabular}{|l|c|}
\hline Gouvernorat & Nombre de ménages \\
\hline Jendouba & 479 \\
\hline Kasserine & 511 \\
\hline Monastir & 472 \\
\hline Nabeul & 501 \\
\hline Total & 1963 \\
\hline
\end{tabular}

Le premier définit l'unité statistique et son identité (gouvernorat, milieu, délégation et district), alors que le second fournit les informations démographiques de base concernant l'âge, le sexe, l'état de résidence et la situation matrimoniale pour tous les membres du ménage et de la famille (décédés ou vivants, présents ou absents). 
Le troisième module porte sur le capital humain, avec des éléments d'information sur le niveau d'éducation, la santé, le niveau de connaissances, ainsi que les compétences. Ce module comporte trois volets : le niveau d'éducation atteint, le travail et les caractéristiques professionnelles de tous les membres âgés de 16 ans et plus, leur profession, leur secteur d'activité, la situation dans la profession (salarié, employeur, etc.), le lieu de travail (public, privé, agricole ou ambulant) et le niveau de compétences. La classification des catégories professionnelles et des compétences est basée sur la Classification internationale CITP (ou ISCO) avec une légère adaptation ${ }^{8}$; nous ajoutons un 5ème niveau et attribuons le niveau 3 aux artisans qui ont pu acquérir des compétences relativement complexes dans certains domaines comme, par exemple, les maîtres-artisans potiers de Nabeul. Dans ce cas, la transmission des compétences se fait à travers des mécanismes traditionnels et familiaux. Ce module fournit aussi des éléments d'information sur le patrimoine physique, la taille des entreprises et des exploitations agricoles où travaillent ou ont travaillé les membres de la famille (y compris les parents, grands-parents et enfants) et leurs principaux produits (ou cultures).

Le module 4, qui porte sur les revenus, se limite au chef de ménage et à son conjoint. Les revenus sont répartis en cinq catégories : revenus du travail, de la propriété, de la pension de retraite, des transferts sociaux et des transferts privés.

Le capital social est l'objet du 5ème module. Les questions posées sont inspirées de la conception présentée dans le cadre théorique qui se base principalement sur les valeurs et les croyances partagées par la société dans son ensemble qui déterminent le niveau de coopération, de cohésion, de civisme et de confiance entre ses membres, faisant du capital social un bien public qui stimule et facilite l'activité économique. Les informations recueillies renseignent sur l'appartenance à des associations ou organisations, sur les personnes auxquelles l'individu est lié et avec qui il coopère, sur la facilité d'obtenir de l'aide auprès de ces personnes, le degré de confiance (ou de méfiance) qui règne entre eux et, directement, sur les valeurs et croyances.

Enfin, le module 6 s'intéresse aux conditions de vie des ménages. Il s'agit d'informations liées aux conditions de logement (type de logement, nombre de pièces, mode d'occupation, mode d'éclairage, gaz naturel, source d'eau potable) et à l'accès aux services sociaux de base, approximé par la distance séparant le logement du jardin d’enfants, école, collège, lycée, centre de santé, hôpital local, établissement de jeunesse, établissement sportif ou centre de commerce ou de services le plus proche.

Le tableau 3 donne la taille et la composition des ménages par groupe d'âge. Par rapport à la taille moyenne des ménages en Tunisie qui s'élève à 4,2 individus, les ménages les plus larges se trouvent dans le gouvernorat de Kasserine avec une moyenne de 4,8, suivi par Monastir avec une moyenne égale à un ménage tunisien typique. Les gouvernorats de Nabeul et de Jendouba regroupent en moyenne des ménages de plus petite taille. Ces chiffres montrent que les arrangements de vie commune diffèrent sensiblement entre le gouvernorat de Kasserine et les autres régions. D'après le même tableau, les ménages de Kasserine semblent plus jeunes que les ménages des trois autres régions, comme en

\footnotetext{
${ }^{8}$ CITP : Classification internationale type des professions ; ISCO : International Standard Classification of Occupations. Cette classification de l'Organisation internationale du travail (OIT) ne fournit que 4 niveaux de compétences, alors que notre travail est basé sur 5 niveaux.
} 
témoigne le nombre moyen des jeunes âgés de moins de 14 ans. La présence de personnes âgées est nettement supérieure dans la région de Jendouba par rapport au reste des régions enquêtées, avec une moyenne de 0,9 individus par ménage, contre 0,6 individus dans chacune des trois autres régions. En effet, un simple test statistique de différence de moyenne entre la région de Jendouba et chacune des trois autres confirme cette hypothèse.

Tableau 3. Composition du ménage par groupe d'âge

Source : calculs des auteurs à partir de l'enquête sur les inégalités en Tunisie, INS 2019

\begin{tabular}{|l|c|c|c|c|c|c|c|c|}
\hline & \multicolumn{2}{|c|}{ Nabeul } & \multicolumn{2}{c|}{ Jendouba } & \multicolumn{3}{c|}{ Monastir } & \multicolumn{2}{c|}{ Kasserine } \\
\hline & Moyenne & Écart-type & Moyenne & Écart-type & Moyenne & Écart-type & Moyenne & Écart-type \\
\hline Taille ménage & 3,7 & 1,4 & 3,7 & 1,8 & 4,2 & 2,0 & 4,8 & 2,1 \\
\hline \# enfants 0-14 ans & 0,7 & 1,0 & 0,6 & 1,1 & 0,8 & 1,2 & 1,1 & 1,5 \\
\hline \# jeunes 15-24 ans & 0,5 & 0,8 & 0,6 & 1,0 & 0,8 & 1,1 & 0,8 & 1,2 \\
\hline \# adultes 25-59 ans & 2,7 & 1,5 & 3,3 & 2,0 & 3,1 & 1,8 & 3,4 & 2,3 \\
\hline \# âgées 60 ans plus & 0,6 & 0,9 & 0,9 & 1,0 & 0,6 & 1,0 & 0,6 & 0,9 \\
\hline
\end{tabular}

Le tableau 4 présente la composition relative des ménages par région enquêtée. Le message principal qui émerge de ces statistiques est que l'arrangement de vie commune diffère sensiblement entre Jendouba et Kasserine, d'une part, et Monastir et Nabeul, d'autre part. Dans les deux premiers gouvernorats, on note la forte présence des parents du chef de ménage, avec une part respective de 2,13 et 2,7\% de l'ensemble des membres vivants dans le ménage, contre seulement 0,94 et 0,8 à Nabeul et à Monastir. Par ailleurs, Monastir et, dans une moindre mesure Kasserine, se distinguent par une forte présence des enfants mariés du chef de ménage, comme en témoigne la part relative des gendres et des belles-filles dans les ménages.

Tableau 4. Composition relative des ménages par gouvernorat (en \%)

Source : calculs des auteurs à partir de l'enquête sur les inégalités en Tunisie, INS 2019

\begin{tabular}{l|ccccc}
\hline & \multicolumn{5}{|c}{ GOUVERNORAT } \\
\cline { 2 - 6 } & Nabeul & Jendouba & Monastir & Kasserine & Total \\
Composition du ménage & & & & & \\
Chef de ménage & 27,41 & 26,90 & 23,74 & 20,90 & 24,41 \\
Conjoint & 22,89 & 20,72 & 18,76 & 16,72 & 19,51 \\
Fils/Fille & 47,93 & 49,19 & 53,52 & 57,34 & 52,46 \\
Petit-fils/fille & 0,50 & 0,56 & 1,66 & 0,98 & 0,95 \\
Gendre/belle-fille & 0,17 & 0,11 & 0,65 & 0,25 & 0,30 \\
Parents & 0,94 & 2,13 & 0,80 & 2,70 & 1,71 \\
Autres & 0,17 & 0,39 & 0,86 & 1,11 & 0,67 \\
Total & 100,00 & 100,00 & 100,00 & 100,00 & 100,00 \\
\hline
\end{tabular}

Le tableau 5 donne l'âge moyen et le niveau d'éducation du chef de ménage. Le niveau d'éducation médian ne varie pas selon la région. La moitié des ménages enquêtés sont dirigés par un individu ayant un niveau primaire ou moins. Concernant l'âge, c'est la région de Jendouba qui se distingue légèrement par l'âge sensiblement plus élevé du chef de ménage. 
Tableau 5. Âge et niveau d'éducation médian du chef de ménage

Source : calculs des auteurs à partir de l'enquête sur les inégalités en Tunisie, INS 2019

\begin{tabular}{|l|c|c|}
\hline & Âge moyen & Niveau d'éducation médian \\
\hline Nabeul & 56,2 & Primaire \\
\hline Jendouba & 61,1 & Primaire \\
\hline Monastir & 55,7 & Primaire \\
\hline Kasserine & 55,7 & Primaire \\
\hline Total & 57,0 & Primaire \\
\hline
\end{tabular}

Le tableau 6 donne l'âge moyen et le niveau d'éducation médian des enfants du chef de ménage. Le niveau d'éducation médian est plus faible dans la région de Kasserine, mais ce résultat est conforme à la composition des ménages mentionnée ci-dessus qui indique une présence plus forte des jeunes de moins de 14 ans dans cette région. Par ailleurs, dans la région de Jendouba l'âge moyen des enfants est nettement supérieur à celui observé dans les autres régions. Encore une fois, ce résultat est conforme aux arrangements de vie commune mentionnés ci-dessus qui révèlent qu'en moyenne les enfants mariés du chef de ménage continent à vivre avec leurs parents.

Tableau 6. Âge et niveau d'éducation des enfants du chef de ménage

Source : calculs des auteurs à partir de l'enquête sur les inégalités en Tunisie, INS 2019

\begin{tabular}{|l|c|c|}
\hline & Âge moyen & Éducation médiane \\
\hline Nabeul & 19,42 & Secondaire \\
\hline Jendouba & 21,92 & Secondaire \\
\hline Monastir & 19,05 & Secondaire \\
\hline Kasserine & 18,06 & Primaire \\
\hline
\end{tabular}

Le niveau d'implication des ménages dans des activités agricoles s'élève à 16\%. En effet, d'après les résultats du tableau 7, le taux d'activité agricole est comparable entres les différentes régions et se situe à près de $12 \%$, à l'exception de la région de Kasserine, où $28 \%$ des ménages exercent une activité agricole.

Tableau 7. Pourcentage des ménages exerçant une activité agricole

Source : calculs des auteurs à partir de l'enquête sur les inégalités en Tunisie, INS 2019

\begin{tabular}{|l|c|}
\hline & Activité agricole en $\%$ \\
\hline & \\
\hline Nabeul & 11,5 \\
\hline Jendouba & 11,9 \\
\hline Monastir & 11,9 \\
\hline Kasserine & 28,4 \\
\hline Total & 16,1 \\
\hline
\end{tabular}




\subsection{Les principaux indicateurs}

Les principaux indicateurs concernent le niveau de vie et de bien-être, le capital humain hérité et le capital social.

\subsubsection{L'indice composite de bien-être (IBE)}

Les inégalités sont habituellement analysées en termes d'inégalités monétaires (de revenus) et non monétaires. Le questionnaire de notre enquête comporte des questions ayant pour but d'obtenir des informations précises sur les différentes sources de revenus du ménage (revenu du travail, transferts et revenus du patrimoine). Malheureusement, les données obtenues n'étaient pas assez précises étant donné que la majorité des personnes interrogées (plus de 90\%) ont préféré donner l'intervalle de gain plutôt que le niveau de leur revenu nominal, et ce selon 10 intervalles variant de 0 à 3500 dinars et plus. Par ailleurs, le revenu hors-travail est donné en terme nominal et non pas par intervalle. Afin d'estimer le revenu rapporté, nous définissons la variable de revenu total comme la somme du centre de l'intervalle déclaré de revenu du travail et le revenu hors-travail déclaré. Toutefois, nous considérons que les données finales sur le revenu ne sont pas assez satisfaisantes et qu'il faut les améliorer en utilisant toutes les informations disponibles sur le niveau de vie des ménages.

L'idée est de calculer un indice composite synthétisant toutes ces informations, que nous appelons indice de bien-être (IBE). L'IBE peut être considéré comme une moyenne pondérée du niveau de revenu et de plusieurs indicateurs élémentaires de conditions de vie ${ }^{9}$. Il s'agit d'une approximation du niveau de vie du ménage qui reflète d'une manière plus fidèle son pouvoir d'achat, ainsi que l'aspect multidimensionnel (monétaire et non monétaire) des inégalités. Nous souhaitons également nous focaliser sur des attributs physiques provenant d'informations robustes et stables qui reflètent le niveau de revenu permanent du ménage. Le Tableau A 4 présente la liste des variables utilisées pour construire l'IBE.

\footnotetext{
9 Quelle que soit la méthode d'analyse factorielle utilisée pour la détermination du poids (analyse en composantes principales ou des correspondances multiples), la forme fonctionnelle de l'indice composite est définie comme suit :

Avec

$$
I C_{i}=\frac{\sum_{k=1}^{K} \sum_{j_{k}=1}^{J_{k}} W_{j_{k}}^{k} I_{i j_{k}}^{k}}{K}
$$

$K$ : le nombre d'indicateurs primaires ;

$J_{k}$ : le nombre de modalités de l'indicateur $\mathrm{k}$;

$W_{j_{k}}^{k}$ : le poids accordé à la modalité $j_{k}$ qui est égal au score de premier axe, obtenu après l'application d'une analyse factorielle à la matrice de données ;

$I_{i j_{k}}^{k}$ : la variable binaire, prenant la valeur 1 lorsque le ménage $i$ a la modalité $j_{k}$, et 0 sinon.

Cet indice sera ensuite standardisé sur une échelle allant de 0 à 1 à l'aide de la méthode Min-Max (en utilisant les valeurs extrêmes de l'échantillon). L'indice composite standardisé sera alors calculé à l'aide de la formule suivante:

$$
I C=\frac{I C_{i}-\min (I C)}{\max (I C)-\min (I C)}
$$
}

À l'issue de la standardisation, la valeur 0 correspondra au niveau le plus faible et 1 au plus élevé. 
L'indice est construit en utilisant une extension de l'analyse en composantes principales (ACP), à savoir l'ACP-polychoriques de Kolenikov et Angeles (2009) qui permet de combiner des données continues, discrètes et ordinales et d'estimer statistiquement le poids de chaque variable.

Notre approche d'approximation du niveau de bien-être du ménage est conforme aux indices de richesse largement utilisés dans les enquêtes MICS ${ }^{10}$ de l'UNICEF pour évaluer les conditions de vie des ménages. La même approche est utilisée, entre autres, dans les enquêtes PISA pour analyser l'équité dans les performances scolaires des enfants.

\subsubsection{L'indice du capital humain hérité ( $\mathrm{CHH})$}

Cet indice permet d'évaluer le stock de capital humain, c'est-à-dire la richesse immatérielle, sous forme de savoir, de savoir-faire et de compétences, transmise par le grand-père en passant par le père, qui l'a lui-même héritée de ses parents et grands-parents. Cet indice reflète aussi le degré de complexité de l'activité ou du métier exercé par les grands-parents. L'indice du capital humain hérité est calculé en utilisant la méthode des correspondances multiples sur la base de trois variables agrégées (voir tableau 8 ) : le niveau d'instruction du grand-père, son niveau de compétence et la complexité de l'activité ou du métier qu'il exerçait. Les deux dernières variables sont codées de 1 à 5 . Il est à noter qu'il pourrait y avoir un biais dans les mesures sur les grands-parents, généré notamment par les erreurs de mémoire dans la collecte des informations portant sur le passé pour des membres décédés depuis longtemps.

Le capital social est mesuré à l'aide des informations données par trois variables qui renseignent sur le niveau de coopération et de confiance qui règne. Ces informations traduisent la perception subjective des personnes ayant répondu qu questionnaire, mais nous supposons qu'une fois agrégées, elles reflètent la qualité des relations sociales, le niveau de cohésion et de confiance entre les membres d'une même communauté.

\footnotetext{
${ }^{10}$ Multiple Indicator Cluster Surveys.
} 
Tableau 8. Indice du capital humain hérité (CHH)

\begin{tabular}{|c|c|}
\hline Variables & Catégories \\
\hline \multirow{8}{*}{$\begin{array}{l}\text { Niveau d'instruction du grand- } \\
\text { père }\end{array}$} & Néant \\
\hline & Primaire \\
\hline & Enseignement de base \\
\hline & Secondaire \\
\hline & Niveau baccalauréat \\
\hline & Formation professionnelle \\
\hline & Technicien/Maîtrise \\
\hline & Ingénieur/Master \\
\hline $\begin{array}{l}\text { Niveau de compétence du } \\
\text { grand-père }\end{array}$ & De 0 à 5 \\
\hline \multirow{12}{*}{$\begin{array}{l}\text { Complexité du secteur } \\
\text { d'activité/métier }\end{array}$} & Administration \\
\hline & Services directs aux particuliers, commerçants et vendeurs \\
\hline & Agriculture, sylviculture et pêche \\
\hline & Exploitants de l'agriculture \\
\hline & Élevage \\
\hline & Forêt et chasse \\
\hline & Métiers qualifiés de l'industrie et de l'artisanat \\
\hline & Métiers qualifiés du bâtiment et assimilés, sauf électriciens \\
\hline & Métiers qualifiés de la métallurgie, de la construction mécanique et assimilés \\
\hline & Métiers qualifiés de l'artisanat et de l'imprimerie \\
\hline & Conducteurs d'installations et de machines, ouvriers de l'assemblage \\
\hline & Professions élémentaires \\
\hline
\end{tabular}




\section{Analyse descriptive}

\subsection{Caractéristiques distributives de l'IBE}

Notre point de départ est l'examen de la distribution de l'IBE. Le panel gauche de la figure 1 montre que la distribution est globalement bimodale reflétant la polarisation de la population interrogée autour de deux moyennes locales de l'IBE. Cette caractéristique semble indiquer que des disparités importantes du niveau de richesse ou de bien-être existent entre les ménages. La polarisation se manifeste aussi sur le plan spatial, comme le montre le panel droit de la figure 1 qui confirme que les ménages des régions de Nabeul et de Monastir sont nettement mieux nantis que ceux de Jendouba et de Kasserine. Ces résultats sont conformes au niveau de dépenses moyennes observées dans ces régions.

\section{Figure 1. Estimation de la densité de l'indice de bien-être}

Source : calculs des auteurs à partir de l'enquête sur les inégalités en Tunisie, INS 2019
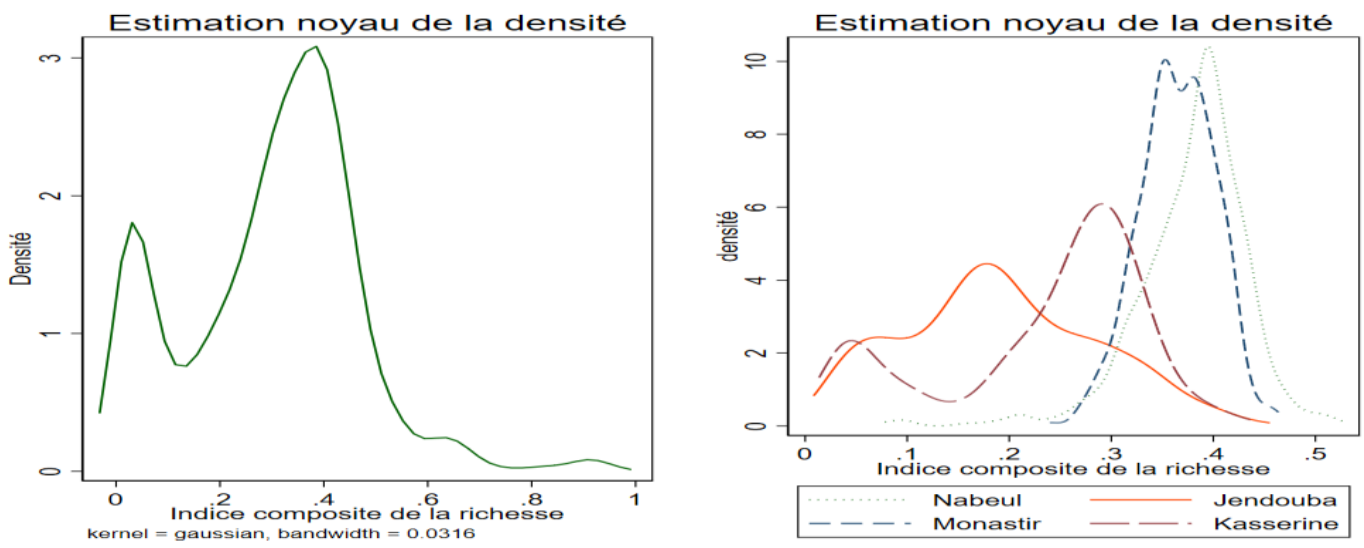

Note : le choix de bandwidth de chaque distribution est basé sur la règle empirique $h=\frac{0.9 m}{\sqrt{n}}$ avec $n$ : nombre d'observations et $m=\min$ (écart type, $\frac{\text { intervalle interquartile }}{1.35}$ ).

Ces résultats sont reflétés également dans le tableau 9 qui présente les statistiques descriptives de l'IBE par région. Le niveau de richesse dans les régions de Nabeul et de Monastir est nettement supérieur à celui observé dans celles de Jendouba et Kasserine. II est aussi important de noter la forte variabilité du niveau de l'IBE dans ces deux dernières régions en comparaison à Nabeul et Monastir. Le coefficient de variation va de 0,71-0,78 dans les deux premières à seulement 0,35-0,37 à Nabeul et à Monastir.

Le niveau de bien-être estimé dans chaque région est conforme aux résultats des enquêtes de consommation des ménages. À titre d'exemple, en 2015 les dépenses moyennes par tête dans les gouvernorats de Monastir, Nabeul, Jendouba et Kasserine s'élèvent respectivement à 5 115, 3 919, 2 943, et 2543 dinars par tête et par an. 
Tableau 9. Statistiques descriptives de l'IBE

Source : calculs des auteurs à partir de l'enquête sur les inégalités en Tunisie, INS 2019

\begin{tabular}{|l|c|c|c|c|c|c|}
\hline & Nb.obs & Moyenne & Écart-type & Min & Max & CV \\
\hline Nabeul & 2251 & 0,35 & 0,12 & 0,00 & 0,91 & 0,34 \\
\hline Jendouba & 2544 & 0,18 & 0,15 & 0,00 & 0,96 & 0,82 \\
\hline Monastir & 2476 & 0,34 & 0,11 & 0,04 & 0,95 & 0,32 \\
\hline Kasserine & 3054 & 0,20 & 0,15 & 0,00 & 0,82 & 0,73 \\
\hline Ensemble & 10325 & 0,26 & 0,15 & 0,00 & 0,96 & 0,58 \\
\hline
\end{tabular}

Pour mieux comprendre la distribution régionale du niveau de vie (tableau 10), nous présentons la distribution des intervalles de revenus salariaux déclarés". Encore une fois, les disparités régionales sont claires et reflètent le même classement de niveau de vie. Plus de $47 \%$ des personnes interrogées (chef de ménage et son époux/épouse) de la région de Jendouba n'ont aucun revenu salarial, contre seulement $25 \%$ pour la région de Nabeul. Toutefois, il est important de noter que ces différences s'expliquent en partie par la nature des activités économiques dans ces régions. Les activités d'auto-emploi dans l'agriculture sont plus répandues dans les gouvernorats de Jendouba et de Kasserine en comparaison avec les autres. Les tableaux tableau 11-13 présentent aussi les conditions d'accès aux services publics et les conditions de logement. Les mêmes disparités apparaissent entre les régions de l'est (Nabeul et Monastir) et de l'ouest (Jendouba et Kasserine), clairement les moins nanties.

Tableau 10. Répartition des intervalles de revenus salariaux

Source : calculs des auteurs à partir de l'enquête sur les inégalités en Tunisie, INS 2019

\begin{tabular}{|l|r|r|r|r|r|}
\hline Intervalle en dinars & Nabeul & \multicolumn{1}{l|}{ Jendouba } & \multicolumn{1}{l|}{ Monastir } & \multicolumn{1}{l|}{ Kasserine } & Total \\
\hline Sans revenu & 25,2 & 47,1 & 36,3 & 42,5 & 37,7 \\
\hline$[0-400]$ & 26,1 & 33,3 & 20,4 & 29,3 & 27,4 \\
\hline$[400-700]$ & 25,1 & 1,0 & 22,6 & 13,1 & 17,9 \\
\hline$[700-1050]$ & 12,6 & 4,9 & 10,4 & 10,4 & 9,6 \\
\hline$[1050-1400]$ & 7,1 & 2,2 & 7,0 & 3,5 & 4,9 \\
\hline$[1400-1750]$ & 2,1 & 1,3 & 1,8 & 1,2 & 1,6 \\
\hline$[1750-2100]$ & 0,7 & 0,1 & 0,9 & 0,0 & 0,4 \\
\hline$[2100-2450]$ & 0,2 & 0,0 & 0,1 & 0,0 & 0,1 \\
\hline$[2450-2800]$ & 0,4 & 0,0 & 0,2 & 0,0 & 0,2 \\
\hline$[2800-3150]$ & 0,1 & 0,0 & 0,1 & 0,0 & 0,1 \\
\hline Plus de 3 150 dinars & 0,4 & 0,1 & 0,1 & 0,0 & 0,2 \\
\hline Total & 100 & 100 & 100 & 100 & 100 \\
\hline
\end{tabular}

11 Ces statistiques concernent les deux conjoints et non pas les ménages. Le nombre d'observations de ce tableau est de 3926. 
Tableau 11. Type de logement selon le gouvernorat

Source : calculs des auteurs à partir de l'enquête sur les inégalités en Tunisie, INS 2019

\begin{tabular}{|l|c|c|c|c|c|}
\hline & Nabeul & Jendouba & Monastir & Kasserine & Total \\
\hline Maison traditionnelle & 5 & 1 & 26 & 6 & 9 \\
\hline Logement jumelé & 74 & 25 & 68 & 58 & 56 \\
\hline Villa & 9 & 15 & 4 & 1 & 7 \\
\hline Étage villa supérieur & 5 & 15 & 1 & 3 & 6 \\
\hline Appartement & 4 & 1 & 1 & 1 & 2 \\
\hline Maison modeste & 3 & 43 & 1 & 32 & 20 \\
\hline Total & 100 & 100 & 100 & 100 & 100 \\
\hline
\end{tabular}

Tableau 12. Gaz naturel selon le gouvernorat

Source : calculs des auteurs à partir de l'enquête sur les inégalités en Tunisie, INS 2019

\begin{tabular}{|l|c|c|c|c|c|}
\hline Gaz naturel & Nabeul & Jendouba & Monastir & Kasserine & Total \\
\hline Oui & 68 & 4 & 22 & 8 & 26 \\
\hline Non & 32 & 96 & 78 & 92 & 74 \\
\hline Total & 100 & 100 & 100 & 100 & 100 \\
\hline
\end{tabular}

Tableau 13. Distance moyenne séparant le logement du plus proche

Source : calculs des auteurs à partir de l'enquête sur les inégalités en Tunisie, INS 2019

\begin{tabular}{|l|c|c|c|c|c|}
\hline & Nabeul & Jendouba & Monastir & Kasserine & \multicolumn{1}{l|}{ Total } \\
\hline École primaire & 88 & 58 & 83 & 68 & 74 \\
\hline Collège & 64 & 35 & 77 & 56 & 58 \\
\hline Lycée & 50 & 23 & 67 & 47 & 47 \\
\hline Centre de santé & 83 & 37 & 79 & 52 & 63 \\
\hline Hôpital & 19 & 15 & 45 & 16 & 23 \\
\hline Crèche & 85 & 44 & 86 & 48 & 66 \\
\hline Établissement pour jeunes & 78 & 29 & 78 & 22 & 51 \\
\hline Établissement sportif & 78 & 29 & 80 & 21 & 52 \\
\hline Centre commercial & 81 & 34 & 77 & 14 & 51 \\
\hline
\end{tabular}


Les tableaux 14-15 présentent la distribution relative de la population par quintile de l'IBE et par région. Plus de $90 \%$ de la population du premier quintile de l'IBE réside dans les régions de Jendouba et Kasserine, alors que le quintile le mieux nanti est formé à hauteur de $77 \%$ d'habitants de Nabeul et de Monastir. ̇̀ Nabeul et Monastir, la majorité des ménages (66\% à Nabeul et $56 \%$ à Monastir) appartiennent aux deux quintiles les plus riches ; à Jendouba et Kasserine, ce taux ne dépasse pas $20 \%$.

Tableau 14. Distribution relative de la population par quintile et par région en $\%$

Source : calculs des auteurs à partir de l'enquête sur les inégalités en Tunisie, INS 2019

\begin{tabular}{|l|c|c|c|c|c|}
\hline & Nabeul & Jendouba & Monastir & Kasserine & Total \\
\hline Q1 IBE & 3 & 37 & 2 & 35 & 21 \\
\hline Q2 IBE & 15 & 31 & 15 & 20 & 20 \\
\hline Q3 IBE & 16 & 12 & 27 & 25 & 20 \\
\hline Q4 IBE & 27 & 12 & 31 & 12 & 20 \\
\hline Q5 IBE & 39 & 9 & 25 & 8 & 19 \\
\hline Total & 100 & 100 & 100 & 100 & 100 \\
\hline
\end{tabular}

Tableau 15. Distribution relative de la population par quintile et par région en \%

Source : calculs des auteurs à partir de l'enquête sur les inégalités en Tunisie, INS 2019

\begin{tabular}{|l|c|c|c|c|c|}
\hline & Nabeul & Jendouba & Monastir & Kasserine & Total \\
\hline Q1 IBE & 4 & 44 & 2 & 51 & 100 \\
\hline Q2 IBE & 16 & 37 & 18 & 29 & 100 \\
\hline Q3 IBE & 17 & 14 & 32 & 37 & 100 \\
\hline Q4 IBE & 30 & 15 & 38 & 18 & 100 \\
\hline Q5 IBE & 45 & 11 & 32 & 12 & 100 \\
\hline Total & 22 & 25 & 24 & 30 & 100 \\
\hline
\end{tabular}

Le tableau 16 montre aussi la forte corrélation entre le niveau d'instruction du chef de ménage et le niveau de bien-être. En effet, près de la moitié de la population du premier quintile vit dans un ménage dont le chef est sans instruction, alors que cette catégorie ne représente que $18 \%$ de la population du dernier quintile. La même remarque s'applique aux autres niveaux d'instruction du chef de ménage.

Tableau 16. Distribution relative de l'IBE par niveau d'instruction du chef de ménage en $\%$

Source : calculs des auteurs à partir de l'enquête sur les inégalités en Tunisie, INS 2019

\begin{tabular}{|l|c|c|c|c|c|}
\hline & Néant & Primaire & Secondaire & Universitaire & Total \\
\hline Q1 IBE & 36,0 & 16,6 & 10,1 & 2,8 & 20,6 \\
\hline Q2 IBE & 25,4 & 19,0 & 17,2 & 12,7 & 20,3 \\
\hline Q3 IBE & 20,0 & 21,3 & 20,3 & 16,0 & 20,3 \\
\hline Q4 IBE & 11,7 & 23,8 & 23,4 & 24,5 & 19,8 \\
\hline Q5 IBE & 7,0 & 19,3 & 29,0 & 44,0 & 19,0 \\
\hline Total & 100 & 100 & 100 & 100 & 100 \\
\hline
\end{tabular}




\subsection{Caractéristiques distributives du $\mathrm{CHH}$}

La figure 2 présente l'estimation de la densité de probabilité du $\mathrm{CHH}$. Les résultats font apparaître une distribution bimodale très similaire à la distribution de l'IBE. Son aspect bimodal est dû aux disparités régionales dans la distribution du $\mathrm{CHH}$, particulièrement ente l'est et l'ouest du pays. En effet, l'examen des statistiques descriptives par région ci-dessus montre que le $\mathrm{CHH}$ est nettement moins important dans les régions de Jendouba et de Kasserine que dans celles de Nabeul et Monastir qui présentent des niveaux similaires de $\mathrm{CHH}$. Le tableau 17 présente les statistiques descriptives du $\mathrm{CHH}$. Il est important de noter en outre la faible variabilité de la distribution du $\mathrm{CHH}$ dans la région de Monastir. Cela peut s'expliquer par la diversité des activités de la région et par l'importance d'activités relativement complexes et plus productives, notamment dans le secteur des textiles et de l'habillement, qui sont répandues dans la région et emploient une partie importante de la population active, voire la majorité dans certaines zones, et ce depuis des siècles. La région de Nabeul est connue également pour ses activités de poterie, relativement complexes du moins par rapport aux activités répandues dans les autres régions de l'ouest du pays. L'estimation présentée révèle clairement une dichotomie entre les régions côtières et celles de l'intérieur du pays en matière d'accumulation du capital humain hérité. Cette corrélation entre la région de résidence et le capital humain hérité sera discutée dans l'explication économétrique des disparités observées du niveau de bien-être. La corrélation entre le $\mathrm{CHH}$ et la région de résidence semble également indiquer qu'il sera important d'analyser de plus près la distribution du $\mathrm{CHH}$ dans les différentes régions du pays dans de futurs travaux. Les enquêtes périodiques conduites par l'INS pourraient intégrer, à un coût raisonnable, cette dimension dans leur questionnaire.

Figure 2. Estimation de la densité du capital humain hérité Source : élaborée par les auteurs à partir de l'enquête sur les inégalités en Tunisie, INS 2019

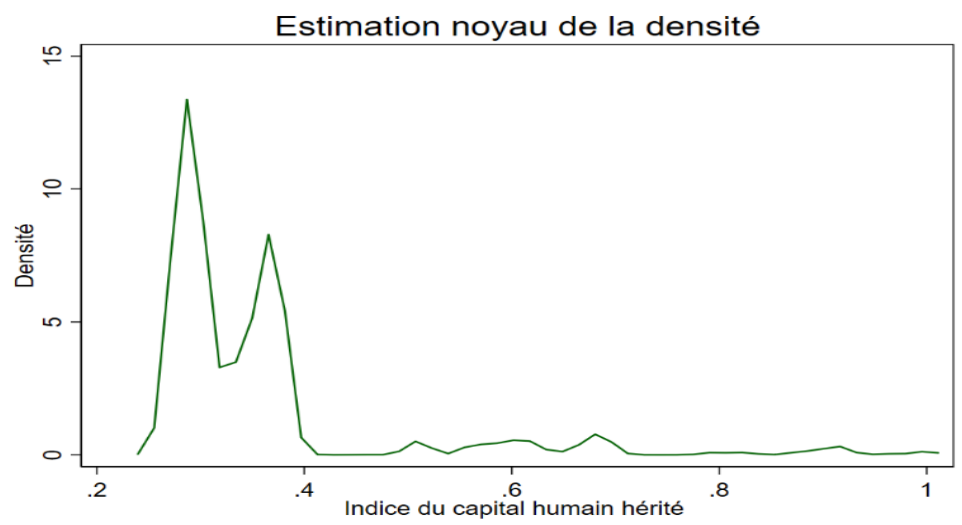

Note : le choix de bandwidth de chaque distribution est basé sur la règle empirique $h=\frac{0.9 m}{\sqrt{n}}$ avec $n:$ nombre d'observations et $m=\min$ (écart type, $\left.\frac{\text { intervalle interquartile }}{1.35}\right)$. 
Tableau 17. Statistiques descriptives du capital humain hérité par région

Source : calculs des auteurs à partir de l'enquête sur les inégalités en Tunisie, INS 2019

\begin{tabular}{|l|c|c|c|c|c|}
\hline & Moyenne & Écart-type & Min & Max & CV \\
\hline Nabeul & 0,34 & 0,22 & 0,00 & 0,98 & 0,66 \\
\hline Jendouba & 0,25 & 0,16 & 0,00 & 1,00 & 0,63 \\
\hline Monastir & 0,36 & 0,16 & 0,00 & 1,00 & 0,45 \\
\hline Kasserine & 0,27 & 0,13 & 0,00 & 1,00 & 0,49 \\
\hline Ensemble & 0,30 & 0,17 & 0,00 & 1,00 & 0,58 \\
\hline
\end{tabular}

Sans surprise, les résultats montrent aussi la forte corrélation entre l'IBE et le $\mathrm{CHH}$, ce qui indique déjà l'importance du $\mathrm{CHH}$ comme facteur explicatif du bien-être et, par suite, des inégalités. En effet, le tableau 18 montre que près de 41 \% de la population du premier quintile du $\mathrm{CHH}$ se retrouve en bas de la distribution de la richesse (les deux premiers quintiles) et près des deux tiers de la population du cinquième quintile du $\mathrm{CHH}$ se retrouve parmi les $40 \%$ les mieux nantis. Le tableau 19 indique que les deux tiers de la population de Nabeul et de Monastir appartiennent aux $4^{\text {ème }}$ et $5^{\text {ème }}$ quintiles, alors que ce taux ne dépasse pas $25 \%$ pour les deux autres.

Tableau 18. Distribution relative de la population par quintile de CHH et IBE en \%

Source : calculs des auteurs à partir de l'enquête sur les inégalités en Tunisie, INS 2019

\begin{tabular}{|l|c|c|c|c|c|c|}
\hline & Q1 CHH & Q2 CHH & Q3 CHH & Q4 CHH & Q5 CHH & Total \\
\hline Q1 IBE & 19,9 & 33,5 & 32,9 & 17,6 & 4,2 & 21,7 \\
\hline Q2 IBE & 21,7 & 27,2 & 20,5 & 20,6 & 16,4 & 20,9 \\
\hline Q3 IBE & 19,5 & 18,8 & 21,3 & 24,0 & 20,0 & 20,8 \\
\hline Q4 IBE & 23,3 & 11,5 & 12,6 & 20,6 & 30,7 & 19,7 \\
\hline Q5 IBE & 15,7 & 9,0 & 12,7 & 17,3 & 28,7 & 17,0 \\
\hline Total & 100 & 100 & 100 & 100 & 100 & 100 \\
\hline
\end{tabular}

Tableau 19. Distribution relative du CHH par région et par quintile en \%

Source : calculs des auteurs à partir de l'enquête sur les inégalités en Tunisie, INS 2019

\begin{tabular}{|l|c|c|c|c|c|}
\hline & Nabeul & Jendouba & Monastir & Kasserine & Total \\
\hline Q1 CHH & 21 & 22 & 8 & 13 & 16 \\
\hline Q2 CHH & 4 & 27 & 8 & 21 & 16 \\
\hline Q3 CHH & 12 & 26 & 15 & 49 & 28 \\
\hline Q4 CHH & 6 & 21 & 36 & 12 & 19 \\
\hline Q5 CHH & 57 & 4 & 33 & 5 & 22 \\
\hline Total & 100 & 100 & 100 & 100 & 100 \\
\hline
\end{tabular}


Dans des termes plus concrets, on voit clairement que dans les régions de Nabeul et de Monastir (voir Tableau A 2 en annexe), 95 \% des grands-pères de tout l'échantillon qui étaient des maîtres artisans (correspondant au niveau de compétence 3) se trouvent à Monastir (57\%) et à Nabeul (38 \%), les 5\% restant étant à Kasserine. Il est à noter que l'âge moyen du chef de famille est autour de 50 ans et que si ses grands-pères étaient encore vivants, ils auraient autour de 110 ans. C'est dire que les grands-pères ont connu la période coloniale et qu'ils étaient porteurs d'un savoir-faire hérité, sachant que la modernisation du pays n'a commencé que plus tard.

De même, la répartition de ces grands-pères par région et par type d'activité exercée (Tableau A 3 en annexe) montre qu'à Nabeul où l'agriculture demeurait en ce temps-là la principale activité, plus de la moitié des agriculteurs (55\%) s'adonnaient à des cultures intensives souvent irriguées, exigeantes en savoir-faire, à savoir le maraîchage et l'arboriculture, y compris des agrumes et de l'olivier. Cette proportion est de $35 \%$ à Monastir, où ce secteur était moins important, et beaucoup plus faible à Jendouba et Kasserine. Dans ces deux dernières régions, comme le confirment les résultats de l'enquête, plus des deux tiers de l'activité agricole des grands-pères (de loin la plus importante) était dans la céréaliculture et l'élevage, activités beaucoup moins intensives et moins complexes. Le même tableau montre qu'à Nabeul et à Monastir, les activités industrielles et artisanales étaient à l'époque des grands-pères déjà importantes : près de 8 \% à Nabeul et de 20 \% à Monastir, alors qu'elles étaient très faibles dans les deux autres régions. Ces activités, quoique basées pour la plupart sur des techniques traditionnelles et plutôt artisanales, étaient tout de même bien plus complexes que la plupart des autres activités de leur temps.

C'est aussi dire à quel point le contexte familial déterminait les compétences et les opportunités offertes aux nouvelles générations. Cette règle n'allait pas disparaître, même si la société tunisienne allait connaître une profonde transformation du pays et la démocratisation de l'éducation à partir des années 1960. On constate que les enfants des familles les plus éduquées sont relativement plus éduqués. La matrice de transition suivante (tableau 20) le confirme, même si elle indique aussi que l'école a offert des chances d'ascension à certains. En effet, l'ascension sociale a permis à plus de la moitié des chefs de famille dont le père n'a pas dépassé le niveau primaire de faire au moins des études secondaires, mais plus de $90 \%$ de ceux dont le père avait fait des études plus avancées ont eux aussi atteint ou dépassé le cap des études secondaires. Les trois quarts des enfants de père ayant fait des études supérieures ont fait de même. Ce mécanisme est plutôt positif, mais il a contribué à la persistance des inégalités sociales et régionales.

Tableau 20. Transition du capital humain pour la population âgée de plus 20 ans (chiffres en \%)

Source : calculs des auteurs à partir de l'enquête sur les inégalités en Tunisie, INS 2019

\begin{tabular}{|l|c|c|c|c|c|}
\hline Éducation du chef de famille & \multicolumn{5}{|c|}{ Éducation du père du chef de famille } \\
\hline Éducation & Néant & Primaire & Secondaire & Universitaire & Total \\
\hline Néant & 22 & 6 & 3 & 1 & 15 \\
\hline Primaire & 36 & 23 & 7 & 2 & 29 \\
\hline Secondaire & 32 & 47 & 46 & 21 & 38 \\
\hline Universitaire & 10 & 23 & 44 & 76 & 19 \\
\hline Total & 100 & 100 & 100 & 100 & 100 \\
\hline
\end{tabular}




\subsection{Caractéristiques distributives du capital social}

Un tableau quelque peu flou concernant le capital social se dégage des résultats de l'enquête dans les quatre régions, ce qui a rendu difficile la construction d'un indice synthétique du capital humain, mais il s'agit tout de même d'un tableau révélateur. Tout d'abord, les Tunisiens dans toutes les régions gardent encore une tendance communautariste qui les pousse à restreindre leurs rapports à leur milieu le plus proche, qui tend à se réduire à la famille. Comme le montre le tableau 21, ce phénomène est encore plus prononcé parmi les couches les moins aisées (premier et deuxième quintiles), mais reste présent même parmi les plus aisés (4 ${ }^{\text {ème }}$ et $5^{\text {ème }}$ quintiles) et les plus instruits. En revanche, l'attachement à une tribu s'est affaibli et ne subsiste que parmi les moins aisés et les moins scolarisés (tableau 23-25), surtout dans les régions de l'ouest (en particulier à Jendouba).

Tableau 21. Distribution relative du groupe sur lequel on compte le plus selon l'IBE (en \%)

Source : calculs des auteurs à partir de l'enquête sur les inégalités en Tunisie, INS 2019

\begin{tabular}{|l|c|c|c|c|c|c|}
\hline & Q1 IBE & Q2 IBE & Q3 IBE & Q4 IBE & Q5 IBE & Moyenne \\
\hline Famille & 97,8 & 93,9 & 87,9 & 89,1 & 86,6 & 90,4 \\
\hline Voisins & 1,9 & 4,6 & 7,7 & 5,1 & 7,6 & 5,7 \\
\hline Collègues, autres & 0,3 & 1,5 & 4,4 & 5,9 & 5,8 & 3,9 \\
\hline Total & 100 & 100 & 100 & 100 & 100 & 100 \\
\hline
\end{tabular}

Tableau 22. Distribution relative du groupe sur lequel on compte le plus en fonction du niveau d'instruction du CM (en \%)

Source : calculs des auteurs à partir de l'enquête sur les inégalités en Tunisie, INS 2019

\begin{tabular}{|l|c|c|c|c|c|}
\hline & Néant & Primaire & Secondaire & Universitaire & Moyenne \\
\hline Famille & 91,3 & 91,3 & 89,1 & 87,6 & 90,4 \\
\hline Voisins & 7,2 & 6 & 4,8 & 1,5 & 5,7 \\
\hline Collègues, autres & 1,5 & 2,7 & 6,1 & 11 & 3,9 \\
\hline Total & 100 & 100 & 100 & 100 & 100 \\
\hline
\end{tabular}

Tableau 23. Appartenance à une tribu : distribution relative selon l'IBE en \%

Source : calculs des auteurs à partir de l'enquête sur les inégalités en Tunisie, INS 2019

\begin{tabular}{|l|c|c|c|c|c|c|}
\hline & Q1 IBE & Q2 IBE & Q3 IBE & Q4 IBE & Q5 IBE & Moyenne \\
\hline Oui & 23 & 20 & 12 & 7 & 6 & 13 \\
\hline Non & 77 & 80 & 88 & 93 & 94 & 87 \\
\hline Total & 100 & 100 & 100 & 100 & 100 & 100 \\
\hline
\end{tabular}


Tableau 24. Appartenance à une tribu : distribution relative selon l'éducation du CM en \%

Source : calculs des auteurs à partir de l'enquête sur les inégalités en Tunisie, INS 2019

\begin{tabular}{|l|c|c|c|c|c|}
\hline & Néant & Primaire & Secondaire & Universitaire & Moyenne \\
\hline Oui & 19 & 12 & 9 & 5 & 13 \\
\hline Non & 81 & 89 & 91 & 95 & 87 \\
\hline Total & 100 & 100 & 100 & 100 & 100 \\
\hline
\end{tabular}

Les tableaux $\mathbf{2 5}$ et $\mathbf{2 6}$ montrent que les valeurs fondamentales (civisme, honnêteté et respect mutuel) semblent très largement partagées par tous et ne pas constituer une ligne de démarcation entre les groupes et les régions. C'est peut-être un bon signe, mais il y a vraisemblablement un grand décalage entre les déclarations et le comportement effectif des individus. Plus généralement, il faut prendre toutes les informations recueillies à ce sujet avec une certaine précaution, d'autant plus que la Tunisie passe par une période instable et historique de transition culturelle marquée par le doute et un certain désarroi.

Néanmoins, la majorité des réponses obtenues indiquent que malgré tout, les Tunisiens continuent, en bonne majorité, à considérer que leurs concitoyens peuvent être dignes de confiance, avec plus ou moins de nuances. Il est en outre remarquable que les régions et les groupes les moins nantis éprouvent moins de méfiance (ou plus de confiance) que les plus nantis. Cela semble déjà indiquer que les inégalités régionales ne s'expliquent pas par la faiblesse relative du capital social des régions défavorisées, contrairement à l'hypothèse de départ.

Tableau 25. Niveau élevé de confiance et d'honnêteté et disposition à coopérer selon la région

Source : calculs des auteurs à partir de l'enquête sur les inégalités en Tunisie, INS 2019

\begin{tabular}{|l|c|c|c|c|c|}
\hline & Nabeul & Jendouba & Monastir & Kasserine & Total \\
\hline Je suis d'accord & 51 & 71 & 47 & 51 & 55 \\
\hline Neutre & 10 & 20 & 39 & 33 & 26 \\
\hline Je ne suis pas d'accord & 39 & 9 & 14 & 16 & 20 \\
\hline Total & 100 & 100 & 100 & 100 & 100 \\
\hline
\end{tabular}

Tableau 26. Niveau élevé de confiance et d'honnêteté et disposition à coopérer selon l'IBE

Source : calculs des auteurs à partir de l'enquête sur les inégalités en Tunisie, INS 2019

\begin{tabular}{|l|c|c|c|c|c|c|}
\hline & Q1 IBE & Q2 IBE & Q3 IBE & Q4 IBE & Q5 IBE & Total \\
\hline Je suis d'accord & 62 & 61 & 55 & 52 & 49 & 55 \\
\hline Neutre & 31 & 23 & 26 & 23 & 25 & 26 \\
\hline Je ne suis pas d'accord & 7 & 15 & 19 & 25 & 26 & 20 \\
\hline Total & 100 & 100 & 100 & 100 & 100 & 100 \\
\hline
\end{tabular}

Quatre individus sur cinq peuvent en outre compter sur la solidarité de leurs proches, la moitié facilement (40\% peuvent facilement emprunter de l'argent en cas de besoin et $38 \%$ avec une certaine difficulté), et cela ne semble pas du tout corrélé avec le niveau de bienêtre des groupes et des régions (tableau 27). 
Tableau 27. Facilité de trouver un prêteur par région

Source : calculs des auteurs à partir de l'enquête sur les inégalités en Tunisie, INS 2019

\begin{tabular}{|l|c|c|c|c|c|}
\hline & Nabeul & Jendouba & Monastir & Kasserine & Total \\
\hline Oui facilement & 38 & 49 & 45 & 31 & 40 \\
\hline Difficilement & 44 & 21 & 37 & 49 & 38 \\
\hline Non impossible & 18 & 31 & 18 & 21 & 22 \\
\hline Total & 100 & 100 & 100 & 100 & 100 \\
\hline
\end{tabular}

Il y a cependant un large consensus que le niveau de confiance a baissé d'une manière notable, un consensus qui prévaut dans toutes les régions et couches sociales (tableau 28 et 29).

Tableau 28. Baisse de la confiance par région

Source : calculs des auteurs à partir de l'enquête sur les inégalités en Tunisie, INS 2019

\begin{tabular}{|l|c|c|c|c|c|}
\hline & Nabeul & Jendouba & Monastir & Kasserine & Total \\
\hline Plus de confiance & 95 & 92 & 94 & 95 & 94 \\
\hline Pas de différence & 4 & 6 & 5 & 2 & 4 \\
\hline Moins de confiance & 2 & 2 & 1 & 3 & 2 \\
\hline Total & 100 & 100 & 100 & 100 & 100 \\
\hline
\end{tabular}

Tableau 29. Baisse de la confiance par IBE

Source : calculs des auteurs à partir de l'enquête sur les inégalités en Tunisie, INS 2019

\begin{tabular}{|l|c|c|c|c|c|c|}
\hline & Q1 IBE & Q2 IBE & Q3 IBE & Q4 IBE & Q5 IBE & Total \\
\hline Plus de confiance & 93 & 95 & 94 & 93 & 94 & 94 \\
\hline Pas de différence & 5 & 3 & 3 & 6 & 4 & 4 \\
\hline Moins de confiance & 2 & 2 & 3 & 1 & 2 & 2 \\
\hline Total & 100 & 100 & 100 & 100 & 100 & 100 \\
\hline
\end{tabular}

\subsection{Le patrimoine physique}

Les données de l'enquête donnent des éléments d'information sur le patrimoine et sur sa transmission à travers l'héritage, un des plus mécanismes de persistance des inégalités les plus importants. Ces données concernent la propriété de la terre et d'entreprises. La matrice suivante (tableau 30) décrit la transition de la terre agricole de père en fils et montre comment la grande majorité de ceux qui sont issus d'une famille sans terre ou de petits propriétaires restent dans cette catégorie, et de même pour ceux issus de propriétaires moyens ou de grands propriétaires. Les termes diagonaux de la matrice sont pour la plupart supérieurs à $75 \%$. Les tableaux $\mathbf{3 1}$ et $\mathbf{3 2}$ décrivent le même mouvement pour la génération précédente et la génération suivante et confirment le même constat, mais avec une certaine dispersion s'expliquant principalement par les règles de l'héritage qui tendent à morceler les terres. 
Tableau 30. Matrice de transition grand-père/père

Source : calculs des auteurs à partir de l'enquête sur les inégalités en Tunisie, INS 2019

\begin{tabular}{|c|c|c|c|c|c|c|c|c|c|}
\hline & & \multicolumn{8}{|c|}{ Père } \\
\hline & & Sar & terre & Moin & de 2 ha & Entre 2 & a et $10 \mathrm{ha}$ & Entre 1 & la et plus \\
\hline \multicolumn{2}{|c|}{ Grand-père } & Irrigué & Pluvial & Irrigué & Pluvial & Irrigué & Pluvial & Irrigué & Pluvial \\
\hline \multirow{2}{*}{ Sans terre } & Irrigué & 92 & 2 & 3 & 0 & 3 & 0 & 0 & 0 \\
\hline & Pluvial & 26 & 61 & 3 & 10 & 0 & 0 & 0 & 0 \\
\hline \multirow{2}{*}{ Moins de $2 \mathrm{ha}$} & Irrigué & 4 & 1 & 90 & 0 & 3 & 1 & 0 & 0 \\
\hline & Pluvial & 6 & 3 & 4 & 84 & 2 & 1 & 0 & 0 \\
\hline \multirow{2}{*}{$\begin{array}{c}\text { Entre } 2 \text { ha et } \\
\text { 10ha }\end{array}$} & Irrigué & 4 & 0 & 12 & 1 & 82 & 1 & 1 & 0 \\
\hline & Pluvial & 3 & 1 & 2 & 10 & 9 & 76 & 1 & 1 \\
\hline \multirow{2}{*}{$\begin{array}{c}\text { Entre 10ha et } \\
\text { plus }\end{array}$} & Irrigué & 0 & 0 & 0 & 0 & 12 & 0 & 88 & 0 \\
\hline & Pluvial & 0 & 1 & 0 & 2 & 4 & 47 & 0 & 45 \\
\hline
\end{tabular}

Tableau 31. Matrice de transition père/fils

Source : calculs des auteurs à partir de l'enquête sur les inégalités en Tunisie, INS 2019

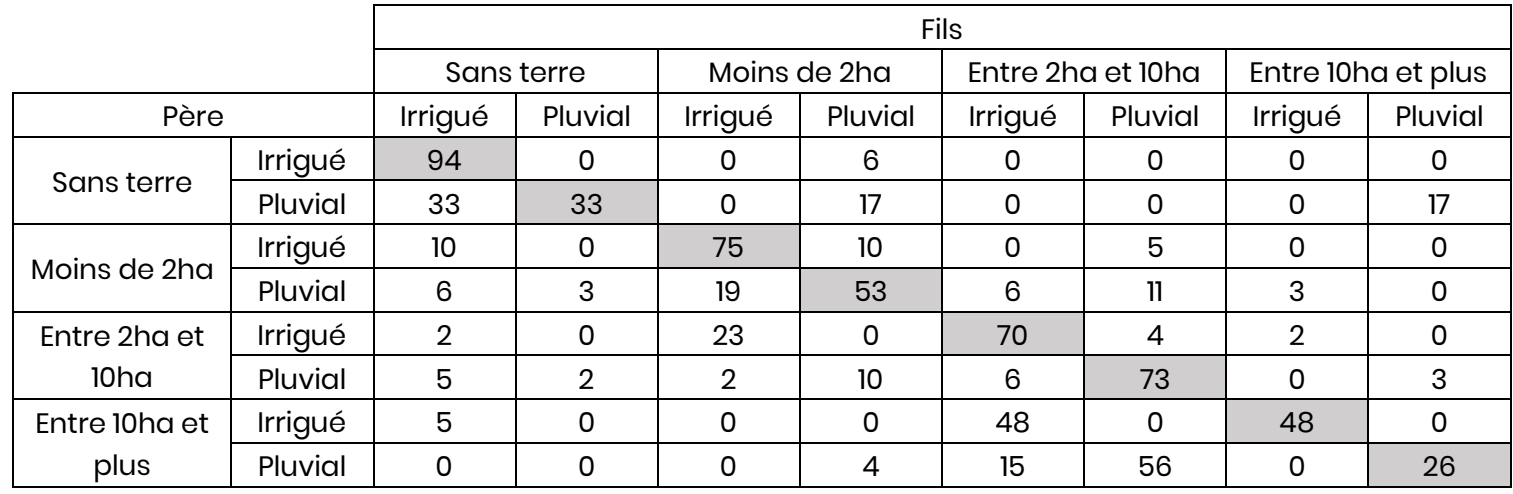

Tableau 32. Matrice de transition grand-père/petit-fils

Source : calculs des auteurs à partir de l'enquête sur les inégalités en Tunisie, INS 2019

\begin{tabular}{|c|c|c|c|c|c|c|c|c|c|}
\hline & & \multicolumn{8}{|c|}{ Petit-fils } \\
\hline & & San & erre & Moins & e 2 ha & Entre 2l & et 10ha & Entre 10 & et plus \\
\hline \multicolumn{2}{|c|}{ Grand-père } & Irrigué & Pluvial & Irrigué & Pluvial & Irrigué & Pluvial & Irrigué & Pluvial \\
\hline \multirow{2}{*}{ Sans terre } & Irrigué & 87 & 0 & 0 & 7 & 7 & 0 & 0 & 0 \\
\hline & Pluvial & 60 & 20 & 0 & 0 & 0 & 0 & 0 & 20 \\
\hline \multirow{2}{*}{ Moins de 2 ha } & Irrigué & 5 & 0 & 71 & 10 & 5 & 10 & 0 & 0 \\
\hline & Pluvial & 6 & 6 & 25 & 38 & 6 & 19 & 0 & 0 \\
\hline \multirow{2}{*}{$\begin{array}{c}\text { Entre } 2 \text { ha et } \\
\text { 10ha }\end{array}$} & Irrigué & 0 & 0 & 23 & 0 & 75 & 0 & 2 & 0 \\
\hline & Pluvial & 15 & 2 & 2 & 8 & 11 & 62 & 0 & 0 \\
\hline \multirow{2}{*}{$\begin{array}{c}\text { Entre 10ha et } \\
\text { plus }\end{array}$} & Irrigué & 5 & 0 & 5 & 0 & 48 & 0 & 43 & 0 \\
\hline & Pluvial & 0 & 0 & 0 & 8 & 6 & 67 & 3 & 17 \\
\hline
\end{tabular}


L'enquête ne fournit qu'une approximation pour mesurer le capital physique. Il s'agit de la taille de l'entreprise où l'individu travaille et de celle du chef de famille qui est aussi chef et propriétaire d'entreprise. Ces entreprises sont codées de 1 à 4 selon leur taille (très petites ou micro, petites, moyennes et grandes). Ces codes sont assimilés à des mesures de la taille du capital de l'entreprise (plus exactement au logarithme de k, le niveau de capital). Pour une personne qui n'est associée à aucune entreprise, $\mathrm{k}=0$. 


\section{Les déterminants des inégalités et du capital social}

\subsection{Le modèle empirique}

Le modèle économétrique est obtenu principalement en linéarisant l'équation (5) résultant du modèle théorique. L'examen des relations de colinéarité entre les variables explicatives révèle une relation entre le capital humain hérité et les indicatrices régionales, d'où l'équation (9).

À côté de ce modèle économétrique de base, on va revenir à l'équation (8) relative à la dynamique du capital social qui ne peut pas être estimée ou calibrée à cause de l'insuffisance des données disponibles, mais qui peut être interprétée et utilisée pour avancer une proposition importante.

Le modèle économétrique se ramène ainsi aux deux équations suivantes :

$$
\begin{gathered}
\mathrm{R}_{\mathrm{i}}=\alpha_{1} \mathrm{k}_{\mathrm{i}}+\alpha_{2} \mathrm{kha}_{\mathrm{i}}+\alpha_{3} \mathrm{khh}_{\mathrm{i}}+\alpha_{4} \mathrm{ks}_{\mathrm{i}}+\alpha_{5} \mathrm{z}_{\mathrm{i}}+\mu_{\mathrm{i}} \\
\mathrm{khh}_{\mathrm{i}}=\beta x_{i}+\mathrm{v}_{\mathrm{i}}
\end{gathered}
$$

Et on a à examiner l'équation dynamique:

$$
\Delta P_{t}=G\left(\Delta N_{t}\right)+g\left(I_{s}, \varepsilon_{t}\right)-\delta P_{t-1}
$$

$\mathrm{R}_{\mathrm{i}}$ est un indicateur de bien-être ou de revenu, $k_{i}, k h a_{i}, k h h_{i}$ et $k s_{i}$ indiquent, respectivement, le niveau du capital physique approximé par la taille de l'entreprise où l'individu $i$ travaille, le niveau de capital humain acquis de cet individu approximé par son niveau d'éducation, le niveau de capital humain hérité de cet individu $i$ et le capital social de sa communauté approximé par sa propre perception subjective du niveau de confiance qui y règne. $z_{i}$ désigne les autres variables explicatives, en premier lieu une indicatrice de la région de l'individu $i$ notée $x_{i}$.

$\mu_{i}, v_{i}$ et $\varepsilon_{t}$ sont des termes aléatoires.

$i=1,2 \ldots, n$, où $n$ est le nombre de ménages couverts par l'enquête.

Ce modèle permet d'étudier l'effet des variables explicatives sur la répartition des revenus $R_{i}$. Une attention particulière sera accordée à l'effet de $k h h, k s$ et l'indicatrice régionale.

$\delta$ indique le taux de dépréciation du capital social, $\Delta N_{t}$ est la variation de $N$ qui peut être provoquée par des mouvements démographiques ou migratoires. Rappelons que $N=$ $\left(n_{0}, n_{1}, n_{2}, \ldots, n_{5}\right)$ donne la répartition des membres de la communauté selon leur degré d'honnêteté et de civisme et détermine le niveau de confiance subjective et donc du capital social qui caractérise cette communauté de $n$ individus. $n=\sum_{s} n_{s}$

Is indique le niveau des investissements publics destinés à l'amélioration du capital social, notamment à travers l'éducation et le maintien de l'État de droit. 


\subsection{Estimation du modèle économétrique}

Pour estimer l'équation relative aux déterminants du revenu, la forme linéaire est acceptable sachant que toutes les grandeurs utilisées pour l'estimer sont en fait des indices que l'on peut assimiler à une approximation du logarithme des variables considérées (log du capital ou du revenu). Par ailleurs, comme nous l'avons fait remarquer dans la partie 3.2 relative à la définition des indicateurs, nous remplaçons le revenu par l'indicateur de bienêtre IBE. Le capital humain acquis ( $k h a$ ) est indiqué par le niveau d'éducation de l'individu. Le capital physique $(k)$ est indiqué par la taille de l'entreprise où est employé l'individu. Enfin, faute d'un indicateur synthétique évident du capital social, nous considérons pour le mesurer deux indicateurs du niveau de confiance subjective exprimé par le chef de famille. Le premier KS1 découle des réponses à la question: "Est-il facile d'emprunter une somme d'argent assez importante auprès d'autre personnes de votre communauté ? " Le second KS2 provient des réponses à la question: "Pensez- vous que les gens ont tellement peu confiance les uns envers les autres qu'ils n'acceptent pas de s'engager entre eux ? "Quand $K S 2$ augmente, cela signifie que l'individu rejette plus cette hypothèse et considère que les gens sont dignes de confiance. La première mesure du capital social s'avère non significative dans toutes les spécifications testées. Nous rapportons dans les résultats ceux relatifs à $K S 2$.

Le tableau 33 présente les résultats d'estimation de trois spécifications différentes. Chaque colonne correspond au résultat d'un modèle. Nous présentons la valeur du coefficient estimé, suivie de la valeur entre parenthèse de l'erreur standard du coefficient, la valeur calculée du T de Student et la p-value.

Le modèle est d'abord estimé en fonction de ( $k h a, k h h, k, K S 2)$, sans aucune autre variable de contrôle (voir le modèle 1). Les résultats confirmant que le capital humain acquis et le capital humain hérité, ainsi que le capital physique, ont bien un effet positif et très significatif sur l'IBE, mais que l'effet du capital social n'est pas significatif, ce qui n'implique pas que le capital social ne soit pas important. L'examen direct des données révèle que certaines couches sociales parmi les plus modestes, notamment dans les régions de l'ouest, ont un capital social plus élevé, car elles expriment plus de confiance et de bienveillance que les autres ; autrement dit, leur IBE (et revenu) plus faible ne s'explique pas par un capital social faible. Toutefois, le peu d'effet du capital social pourrait résulter d'une erreur de mesure et de son contenu subjectif, rendant l'estimation moins précise. Comme mentionné dans la revue de la littérature, Gennaioli et al. (2013) trouvent aussi que la confiance n'a pas d'effet significatif.

Le modèle a ensuite été estimé en introduisant des indicatrices (dummies) pour détecter l'effet spécifique des régions : une par région, avec la région de Nabeul comme référence. II s'est alors révélé que les variables indicatrices des régions de Jendouba et Kasserine (l'ouest du pays le moins nanti) sont significatives avec un signe négatif, ce qui confirme que le niveau de richesse dans ces régions est moins important même lorsque nous contrôlons par les autres caractéristiques individuelles. Les résultats du modèle 2 montrent qu'il n'y a pas une différence statistiquement significative entre la région de Nabeul et celle de Monastir. Dans cette même deuxième spécification, le coefficient du capital humain hérité demeure significatif, mais à un niveau moindre (niveau de significativité à 7,4\%). 
Cette perte relative de significativité s'explique par la forte relation entre la région et $k h h$, ce qui semble indiquer que $k h h$ et les indicatrices rendent compte dans une certaine mesure d'effets similaires et que la spécificité de la région réside en bonne partie dans la différence en matière de $k h h$.

La troisième spécification (modèle 3 dans le tableau 33) utilise une variable indicatrice de l'est par opposition à l'ouest du pays. Les conclusions sont similaires qualitativement au modèle 2. L'existence d'une forte relation entre le $k h h$ et les indicatrices des régions n'est pas surprenante, comme nous l'avons vu. Elle est confirmée par les coefficients significatifs de la régression de $k h h$ sur les indicatrices des régions.

Certaines variables n'ont pas été prises en considération à cause du manque de données, telles que l'accès au financement, la structure et la qualité des institutions, les infrastructures publiques.

Dans un second temps, nous considérons une forme non linéaire du modèle. Nous estimons ainsi un modèle logit ordonné des cinq quintiles de l'IBE. Ce modèle permet d'estimer la probabilité d'être dans un quintile donné. Le sens de corrélation entre l'ordre de quintile et une variable explicative signifie une corrélation dans le même sens entre le niveau de revenu et la variable en question. Le tableau $\mathbf{3 4}$ présente les résultats des estimations de deux spécifications basées sur le logit ordonné. Les variables seuils (seuill-seuil4) représentent en quelque sorte les constantes du modèle (4 seuils pour franchir les 5 quintiles). Les résultats montrent une corrélation positive et significative entre le capital humain hérité et la probabilité d'être en haut de la distribution de revenu. Par ailleurs, dans ce modèle non linéaire, les résultats révèlent une faible corrélation positive entre le capital social et le quintile de revenu (coefficient significatif à 10\%).

Du point de vue qualitatif, quel que soit le modèle utilisé, les conclusions sont similaires. Ainsi, les différentes méthodes d'estimation et spécifications testées confirment que le capital humain hérité explique d'une manière très significative les écarts de niveau de bien-être et donc les inégalités sociales et régionales. En revanche, le capital social demeure non (ou peu) significatif, ce qui est en parfaite cohérence avec les résultats obtenus directement à l'aide des statistiques descriptives.

Le message qui émerge de l'ensemble des analyses économétriques et descriptives des données de l'enquête est le suivant. Indépendamment du rôle incontestable de l'infrastructure et du capital humain acquis, les circonstances individuelles mesurées ici par le capital humain hérité jouent un rôle significatif dans la détermination des revenus. Ce résultat a de fortes implications en matière de politiques économiques qui seront discutées dans la conclusion. II est clair que le rôle des politiques publiques ne devrait pas se limiter à une réponse aux besoins de l'infrastructure (tangibles et intangibles) ou à la facilitation de l'accumulation du capital humain. 
Tableau 33. Résultats d'estimation des spécifications linéaires de l'IBE

Source : calculs des auteurs à partir de l'enquête sur les inégalités en Tunisie, INS 2019

\begin{tabular}{|c|c|c|c|}
\hline & Modèle 1 & Modèle 2 & Modèle 3 \\
\hline Éducation & $0,112^{* * *}$ & $0,077^{* * *}$ & $0,080 * * *$ \\
\hline Erreur standard & $(0,010)$ & $(0,009)$ & $(0,009)$ \\
\hline Stat- $T$ & 11,137 & 8,371 & 8,647 \\
\hline$p$-value & $(0,000)$ & $(0,000)$ & $(0,000)$ \\
\hline Capital physique & $0,024^{* * *}$ & $0,037^{* * *}$ & $0,035^{* * *}$ \\
\hline Erreur standard & $(0,007)$ & $(0,006)$ & $(0,007)$ \\
\hline Stat- $T$ & 3,432 & 5,761 & 5,430 \\
\hline$p$-value & $(0,001)$ & $(0,000)$ & $(0,000)$ \\
\hline Capital social & 0,028 & 0,012 & 0,003 \\
\hline Erreur standard & $(0,017)$ & $(0,015)$ & $(0,015)$ \\
\hline Stat-T & 1,623 & 0,797 & 0,226 \\
\hline$p$-value & $(0,105)$ & $(0,425)$ & $(0,821)$ \\
\hline $\mathrm{CHH}$ & $0,307^{* * *}$ & $0,082^{*}$ & $0,097^{* *}$ \\
\hline Erreur standard & $(0,052)$ & $(0,046)$ & $(0,046)$ \\
\hline Stat- $T$ & 5,942 & 1,789 & 2,125 \\
\hline$p$-value & $(0,000)$ & $(0,074)$ & $(0,034)$ \\
\hline Jendouba & & $-0,328 * * *$ & \\
\hline Erreur standard & & $(0,021)$ & \\
\hline Stat-T & & $-15,324$ & \\
\hline$p$-value & & $(0,000)$ & \\
\hline Monastir & & 0,012 & \\
\hline Erreur standard & & $(0,016)$ & \\
\hline Stat- $T$ & & 0,764 & \\
\hline$p$-value & & $(0,445)$ & \\
\hline Kasserine & & $-0,250 * * *$ & \\
\hline Erreur standard & & $(0,021)$ & \\
\hline Stat-T & & $-11,899$ & \\
\hline$p$-value & & $(0,000)$ & \\
\hline Est & & & $0,290 * * *$ \\
\hline Erreur standard & & & $(0,015)$ \\
\hline Stat-T & & & 18,807 \\
\hline$p$-value & & & $(0,000)$ \\
\hline Constant & $0,230 * * *$ & $0,498 * * *$ & $0,209 * * *$ \\
\hline Erreur standard & $(0,031)$ & $(0,030)$ & $(0,027)$ \\
\hline Stat- $T$ & 7,425 & 16,793 & 7,643 \\
\hline$p$-value & $(0,000)$ & $(0,000)$ & $(0,000)$ \\
\hline Observations & 1558 & 1558 & 1558 \\
\hline $\mathbf{R 2}$ & 0,143 & 0,327 & 0,320 \\
\hline \multicolumn{4}{|c|}{ *** $p<0,01,{ }^{* *} p<0,05,{ }^{*} p<0,1$} \\
\hline
\end{tabular}


Tableau 34. Résultats d'estimation du modèle Logit ordonné

Source : calculs des auteurs à partir de l'enquête sur les inégalités en Tunisie, INS 2019

\begin{tabular}{|c|c|c|}
\hline & Modèle 4 & Modèle 5 \\
\hline Éducation & $0,618^{* * *}$ & $0,497^{* * *}$ \\
\hline Erreur standard & $(0,061)$ & $(0,064)$ \\
\hline Stat-T & 10,104 & 7,709 \\
\hline$p$-value & $(0,000)$ & $(0,000)$ \\
\hline Capital & 0,063 & $0,155^{* * *}$ \\
\hline Erreur standard & $(0,041)$ & $(0,043)$ \\
\hline Stat-T & 1,527 & 3,562 \\
\hline$p$-value & $(0,127)$ & $(0,000)$ \\
\hline Capital social & 0,305 & $0,162 *$ \\
\hline Erreur standard & $-0,19$ & 0,098 \\
\hline Stat-T & 1,6 & 1,653 \\
\hline$p$-value & $-0,11$ & $-0,1$ \\
\hline CHH & $2,143^{* * *}$ & $1,109 * * *$ \\
\hline Erreur standard & $(0,319)$ & $-0,322$ \\
\hline Stat-T & 6,727 & 3,445 \\
\hline$p$-value & $(0,000)$ & $(0,001)$ \\
\hline Est & & $1,859 * * *$ \\
\hline Erreur standard & & $(0,110)$ \\
\hline Stat-T & & 16,944 \\
\hline$p$-value & & $(0,000)$ \\
\hline Seuil 1 & $1,030 * * *$ & $1,290 * * *$ \\
\hline Erreur standard & $(0,192)$ & $(0,195)$ \\
\hline Stat-T & 5,367 & 6,605 \\
\hline$p$-value & $(0,000)$ & $(0,000)$ \\
\hline Seuil 2 & $1,995^{* * *}$ & $2,410 * * *$ \\
\hline Erreur standard & $(0,192)$ & $(0,202)$ \\
\hline Stat- $T$ & 10,403 & 11,911 \\
\hline$p$-value & $(0,000)$ & $(0,000)$ \\
\hline Seuil 3 & $2,861^{* * *}$ & $3,439 * * *$ \\
\hline Erreur standard & $(0,197)$ & $(0,208)$ \\
\hline Stat- $T$ & 14,544 & 16,520 \\
\hline$p$-value & $(0,000)$ & $(0,000)$ \\
\hline Seuil 4 & $3,830 * * *$ & $4,555^{* * *}$ \\
\hline Erreur standard & $(0,208)$ & $(0,224)$ \\
\hline Stat- $T$ & 18,424 & 20,313 \\
\hline$p$-value & $(0,000)$ & $(0,000)$ \\
\hline Observations & 1558 & 1558 \\
\hline \multicolumn{3}{|c|}{${ }^{* * *} p<0,01,{ }^{* *} p<0,05,{ }^{*} p<0,1$} \\
\hline
\end{tabular}

Toute intervention publique devrait aussi tenir compte des circonstances individuelles et de la capacité (telle que reflétée par le capital humain hérité) à saisir les opportunités économiques offertes. 
Enfin, il est important de reconnaître les limites de l'analyse présentée dans ce papier. Tout d'abord, l'enquête n'est pas représentative de l'ensemble de la population. Il serait important dans le futur de confirmer les résultats à l'aide d'une enquête de grande envergure. Nous avons effectué des tentatives de mesure du capital social, mais il faudrait développer d'autres mesures afin de mieux analyser son rôle dans la détermination du revenu.

En outre, nous constatons que le résultat pour le capital social pourrait également être dû à ses mesures subjectives qui ne semblent pas assez robustes. II sera donc intéressant à l'avenir d'approfondir l'analyse de différentes définitions plus robustes pour établir l'effet du capital social sur les inégalités de revenu en Tunisie. Par ailleurs, les difficultés rencontrées lors de cette enquête pour mesurer le revenu des ménages soulèvent la question de la capacité du système statistique tunisien à collecter des informations sur les revenus. À ce jour, aucune enquête menée par l'INS ne contient une telle information (le revenu selon ses différentes sources). L'introduction de modules relatifs aux revenus et au capital (sous ses différentes formes) dans les enquêtes régulières menées par l'INS pourrait offrir de nouvelles opportunités pour l'analyse des déterminants du développement économique et des disparités de revenu.

\section{Analyse de robustesse des résultats}

L'une des potentielles faiblesses de ce travail est l'utilisation d'un indicateur synthétique de bien-être qui reflète les conditions de vie des ménages. Afin de donner plus de robustesse aux résultats précédents, nous présentons dans ce qui suit quelques éléments d'analyse des disparités basés sur les données de l'enquête sur le revenu. Rappelons que le revenu rapporté par le ménage est estimé en additionnant le centre de l'intervalle de revenu déclaré par le ménage, dont les transferts et autres revenus.

En examinant les statistiques de l'enquête sur le revenu mensuel par tête estimé (tableau 35) ou la densité par région (figure 3), les mêmes disparités mentionnées ci-dessus apparaissent. On constate une nette distinction entre d'une part les régions de Nabeul et de Monastir et d'autre part celles de Jendouba et Kasserine. Notons aussi que le revenu par tête est supérieur à la moyenne connue dans les différentes régions. Ce phénomène est essentiellement dû à l'imputation du centre de l'intervalle de revenu déclaré au ménage. Cette imputation n'altère toutefois pas les conclusions relatives aux disparités régionales.

Tableau 35. Statistiques descriptives de revenu mensuel par tête en TND

Source : calculs des auteurs à partir de l'enquête sur les inégalités en Tunisie, INS 2019

\begin{tabular}{|l|c|c|c|c|}
\hline & Moyenne & Écart-type & $\min$ & $\max$ \\
\hline Jendouba & 364,95 & 569,82 & 30,77 & 6800 \\
\hline Kasserine & 356,49 & 885,15 & 30 & 17306 \\
\hline Monastir & 385,54 & 364,82 & 30 & 3217 \\
\hline Nabeul & 538,65 & 790,78 & 33,33 & 14500 \\
\hline
\end{tabular}


Figure 3. Estimation de la densité de revenu mensuel par tête

Source : élaborée par les auteurs à partir de l'enquête sur les inégalités en Tunisie, INS 2019

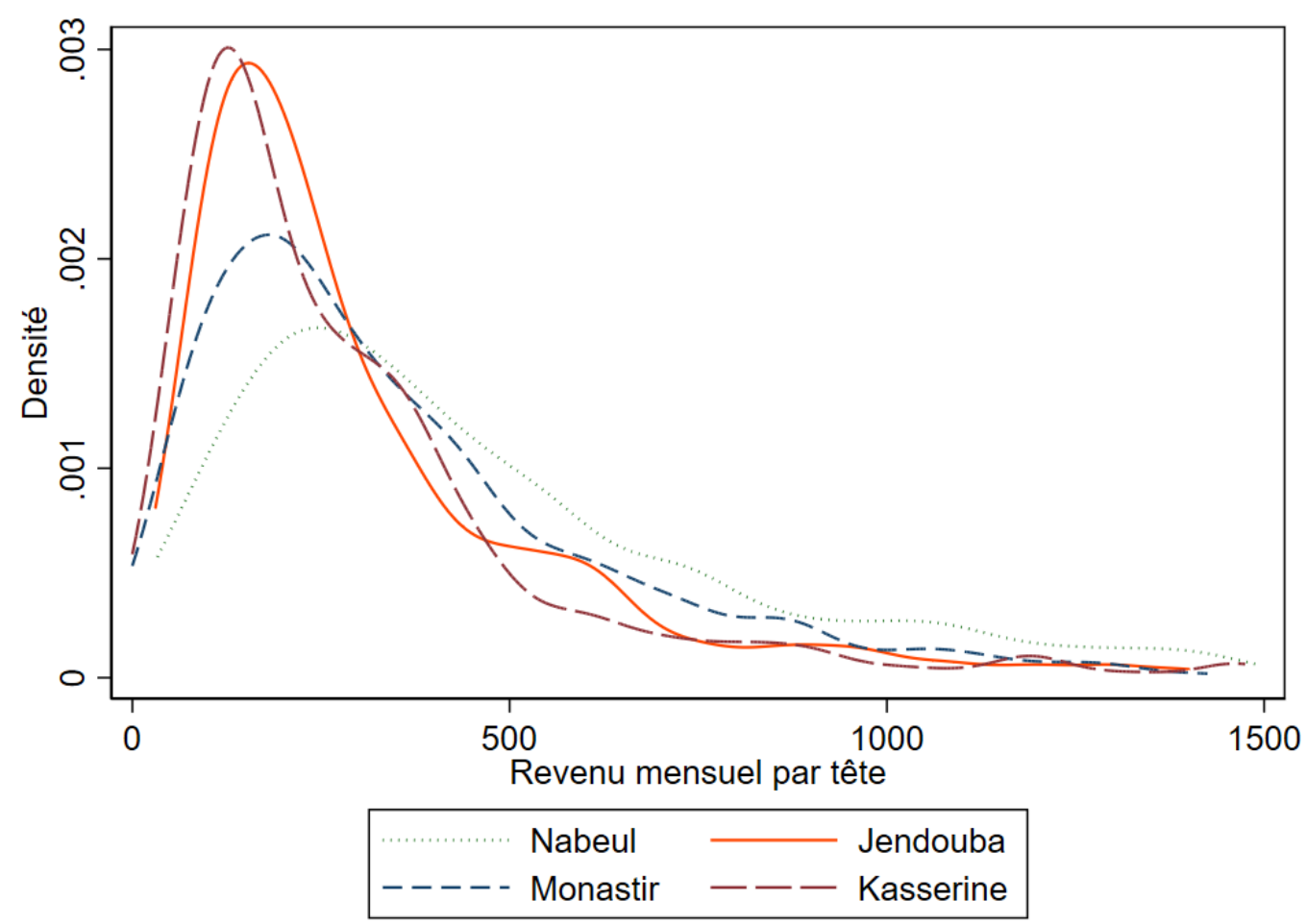

Tableau 36. Régression du log de revenu par tête

Source: calculs des auteurs à partir de l'enquête sur les inégalités en Tunisie, INS 2019

\begin{tabular}{|l|c|c|}
\hline & Modèle I & Modèle II \\
\hline Capital humain hérité & $0,355^{* * *}$ & $(0,131)$ \\
\hline & $(0,128)$ & $-0,02$ \\
\hline Capital social & $-0,021$ & $(0,05)$ \\
\hline & $(0,051)$ & $(0,061)$ \\
\hline Primaire & $0,450^{* * *}$ & $0,773^{* * *}$ \\
\hline & $(0,062)$ & $(0,064)$ \\
\hline Secondaire & $0,812^{* * *}$ & $1,415^{* * *}$ \\
\hline & $(0,065)$ & $(0,083)$ \\
\hline Universitaire & $1,482^{* * *}$ & $0,066^{* * *}$ \\
\hline & $(0,086)$ & $(0,019)$ \\
\hline Capital physique & $0,057^{* * *}$ & $-0,286^{* * *}$ \\
\hline & $(0,019)$ & $(0,056)$ \\
\hline Jendouba & & $-0,367^{* * *}$ \\
\hline & & $(0,057)$ \\
\hline Monastir & & $-0,481^{* * *}$ \\
\hline & & $(0,061)$ \\
\hline Kasserine & & $5,018^{* * *}$ \\
\hline & & $(0,158)$ \\
\hline Constant & $(0,139)$ & 0,269 \\
\hline & 0,231 & \\
\hline R-squared & & \\
\hline
\end{tabular}


Le tableau 36 donne les résultats de la régression du logarithme du revenu par tête estimé du ménage en fonction des variables d'intérêt. Les résultats confirment les principaux messages développés dans ce papier, à savoir l'importance du capital humain hérité dans l'explication des disparités entre les individus et les régions, en contrôlant par les différences en matière de capital humain acquis. Ces résultats confirment aussi que le capital social n'a pas d'effet statistiquement significatif sur les disparités.

Une autre manière de montrer l'effet positif du KHH consiste à examiner la fonction de densité cumulative pour deux groupes d'individus. Le premier regroupe les individus ayant un $K H H$ inférieur à la médiane observée et le second ceux ayant un $K H H$ supérieur à la médiane. Les résultats présentés dans la figure $\mathbf{4}$ montrent la dominance de la courbe du second groupe, particulièrement parmi les individus du second groupe. Le $\mathrm{KHH}$ joue plus particulièrement un rôle discriminant pour les individus ayant un faible revenu.

Figure 4. Fonction de densité cumulative de revenu en fonction du capital humain hérité Source : élaborée par les auteurs à partir de l'enquête sur les inégalités en Tunisie, INS 2019

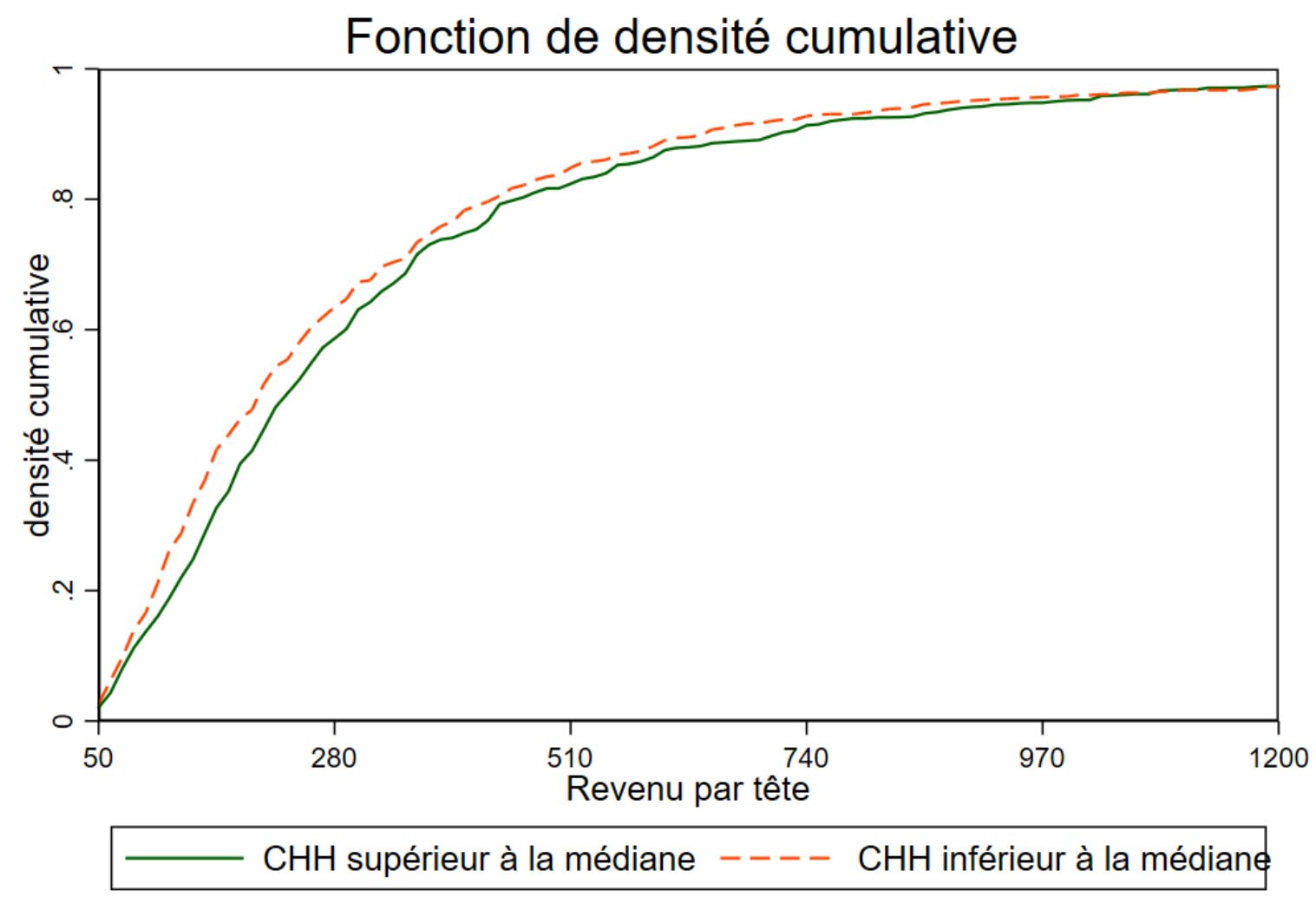




\section{3. Évolution du capital humain : interprétation de l'équation dynamique}

$$
\Delta P_{t}=G(\Delta N t)+g\left(I s, \varepsilon_{t}\right)-\delta P_{t-1}
$$

Comme nous l'avons vu ci-dessus (tableau 28), une très forte majorité des Tunisiens interrogés dans les quatre régions considère que le niveau de confiance a baissé par rapport à l'époque de leurs parents, donc que le capital social se détériore, d'où : $\Delta P_{t}<0$

Quelles hypothèses pour expliquer cette baisse du capital social ?

Partant de l'équation (8), deux hypothèses principales méritent d'être envisagées, en plus de la dépréciation naturelle du capital social :

a) Hypothèse de l'effet des flux migratoires: $G(\Delta N t)<0$

b) Faiblesse d'Is : $g\left(I s, \varepsilon_{t}\right)<0$

La première hypothèse concerne la première composante de l'équation (8), $G(\Delta N t)$, qui stipule que la détérioration du capital social pourrait provenir de l'impact de la variation de la structure démographique $(\Delta N t)$ engendrée par les flux migratoires que le pays a connus au cours des décennies qui ont suivi l'indépendance (depuis 1956). Cette hypothèse est rejetée d'abord parce que la baisse ne se limite pas aux régions d'accueil des migrants (les zones côtières). En outre, le niveau du capital social dans les régions d'origine des migrants, l'intérieur du pays, ne semble pas inférieur à celui des régions d'accueil ; l'inverse semble plus plausible, si bien que la migration ne peut pas être à l'origine de la baisse du capital social ; elle pourrait même l'améliorer. On peut donc écarter cette hypothèse.

La deuxième hypothèse porte sur la faiblesse d'/s, c'est-à-dire de l'investissement dans le capital social, donc de l'investissement dans le système éducatif et le renforcement de l'autorité de l'État. Par élimination, c'est l'hypothèse la plus plausible. Malgré les avancées dans le domaine de l'éducation que la Tunisie a réalisées depuis les années 1960, il existe une défaillance notoire en matière d'apprentissage du civisme et des valeurs favorables à la cohésion sociale, peut-être plus au cours des deux dernières décennies. En même temps, la détérioration de l'État de droit et du respect de la loi et de la justice est de nature à nourrir et à propager plus de méfiance. Cette proposition est plus une conjecture qu'une conclusion ferme et elle mérite des recherches et une analyse plus approfondies. Elle semble néanmoins indiquer que la réforme du système éducatif en cours de préparation devrait accorder une plus grande importance à cette dimension fondamentale. 


\section{Conclusion}

Le principal résultat de ce travail réside dans la confirmation de l'importance du capital humain hérité et la transmission intergénérationnelle du patrimoine matériel et immatériel comme facteurs explicatifs des inégalités, notamment entre les régions. Évidemment, ces facteurs viennent s'ajouter aux autres facteurs connus. Cependant, l'incertitude n'est pas levée concernant le capital social.

Le résultat concernant le capital humain hérité a des implications concrètes en matière de politiques publiques. II sera important de le prendre systématiquement en considération dans la conception et la planification du développement et des investissements publics. On ne peut introduire et développer avec succès de nouvelles activités ou industries dans une région donnée sans en tenir compte et sans prévoir des mesures de formation et de mise à niveau des populations concernées.

Le potentiel de développement d'une région donnée (ou d'un territoire plus ou moins étendu) dépend moins de ses ressources naturelles et de son emplacement géographique que de son potentiel humain, résultat de son patrimoine hérité en matière de capital humain (savoir et savoir-faire) et du capital humain récemment acquis et produit par le système éducatif. Dans la mesure du possible, il est opportun de fonder le développement sur la base du capital humain hérité de chaque région. Autrement dit, en cherchant à identifier le potentiel de chaque région, il est important de voir non seulement ses ressources naturelles (comme on le fait dans les schémas directeurs de l'aménagement du territoire tunisien), mais aussi d'explorer et de valoriser son patrimoine historique. Les régions de l'ouest tunisien ne manquent pas de savoir-faire spécifique, mais il est mal connu et sous-exploité. Plus particulièrement, les femmes de ces régions maîtrisent des techniques artisanales diverses souvent sous-estimées. Si ces femmes pouvaient accéder aux moyens nécessaires (formation et financement), elles pourraient faire preuve de capacités entrepreneuriales et contribuer à l'accélération du développement de leur région.

Toutefois, sachant que certaines régions disposent de ressources matérielles importantes pour développer de nouvelles activités et industries et pour créer de véritables clusters, mais ont un capital humain hérité insuffisant, il est essentiel de les préparer et de les mettre à niveau sur le plan des ressources humaines. Par exemple, là où on estime que les ressources naturelles justifient le développement de nouvelles industries de matériaux de construction et d'industries agroalimentaires associées à lintensification et la modernisation de zones agricoles, alors que la population n'a pas de tradition dans ces domaines et n'y est pas préparée, il faudrait concomitamment investir plus dans la formation, conformément aux besoins des projets de développement envisagés.

Il en découle des implications concrètes concernant le système de formation et d'éducation qui devrait être remodelé et réorienté en fonction des nouveaux besoins de l'économie, lesquels dépendent des particularités de chaque région. 
Le système éducatif est aussi appelé à consacrer plus d'attention et de moyens à l'éducation civique et à la dissémination des valeurs socialement bénéfiques qui constituent l'ossature du capital social. Selon nos analyses préliminaires, le capital social s'est détérioré au cours des dernières décennies parce que le système éducatif ne semble pas assurer son rôle dans le domaine du renforcement des valeurs et du civisme.

L'effet peu significatif du capital social sur la répartition des revenus et les inégalités indique une indétermination plutôt qu'un résultat définitif et robuste. Par définition, le capital social doit être un facteur déterminant des revenus et donc de la répartition et des inégalités des revenus. Les résultats que nous avons obtenus sur la base des données de l'enquête soulèvent plus d'interrogations qu'ils n'apportent de réponses précises. Ils montrent que le capital social n'est pas plus fort dans les régions côtières et semblent indiquer que les Tunisiens tissent leurs relations dans des communautés trop étroites qui tendent à se réduire à leur milieu familial, ce qui révèle une faiblesse structurelle du capital social et remet en question les indicateurs utilisés pour le mesurer.

L'effet peu significatif du capital social pourrait être dû aux mesures du capital social analysées qui ne semblent pas assez robustes. Le capital social devrait donner lieu à des recherches plus approfondies, car le sujet est loin d'être anodin. ̀̀ l'avenir, il conviendrait d'approfondir l'analyse des différentes définitions pour mieux identifier l'effet du capital social sur les inégalités de revenus en Tunisie. L'enquête conduite dans le cadre de cette étude est la première de son genre dans le pays et ne constitue qu'un premier pas. II est également souhaitable de mettre en place des enquêtes auprès des entreprises qui font face plus directement et plus concrètement aux risques associés à la faiblesse du capital social. De même, linsuffisance des informations sur les revenus reste une contrainte de taille qu'il faudra essayer de surmonter par le biais d'autres enquêtes et investigations.

Les résultats obtenus ne minimisent en rien l'importance des autres facteurs de développement (les infrastructures, les institutions, l'accès au financement, etc.) qui n'ont pas été explicitement pris en considération à cause du manque de données fines les concernant, mais qui sont évoquées dans la revue de la littérature et traitées dans Boughzala et al. (2020).

L'histoire est importante, mais elle n'est pas une fatalité. 


\section{Bibliographie}

Acemoglu, D. (2003).

Technology and Inequality.

NBER Reporter: Winter 2003,

https://data.nber.org/reporter/

winter03/technologyandinequa

lity.html

Acemoglu, D. \& Melissa, D.

(2009). Productivity Differences Between and Within Countries. NBER Working Papers 15155 , National Bureau of Economic Research, Inc.

Assaad, R. \& Boughzala, M. (2018). The Tunisian Labor Market in an Era of Transition. Oxford University Press, 2018.

Barro, R. (1991). Economic Growth in a Cross-Section of Countries. Quarterly Journal of Economics, 106(2), pp.407-443.

Becker, G S. (1964). Human capital, A theoretical and empirical analysis with special reference to education, Columbia University Press 1964

Boughzala,M., El Lahga, A. Bouassida, I. et Ferjani, $M$. (2020). Les inégalités en Tunisie. Papiers de recherche AFD n 144 , AFD.

Bourdieu, P. (1985). The genesis of the concepts of habitus and field. Sociocriticism 1(2), pp.l1-24.

Bourguignon, F. (2016). The globalization of Inequality, Princeton and Oxford, Princeton University Press.

Coleman, J. S. (1990).

Foundations of Social Theory. Cambridge, MA: The BelknapoPress of Harvard University Press.

Cook, K. S. (2014). Social Capital and Inequality: The Significance of Social Connections, in "Handbook of the social Psychology of inequality"

Djaït, H., Talbi,M., Dachraoui,F., Dhouib, A., M'rabet, M.A. et Mahfoudhi, F. (2015). Histoire Générale de la Tunisie : Le Moyen-Âge. Sud Éditions 2015.

Dufur, M. J., Parcel, T. L., et Troutman, K. (2013). Does capital at home matter more than capital at school? Social capital effects on academic achievement. Research in Social Stratification and Mobility, 31, pp.1-21.

\section{Fuente, A. et R. Doménech} (2006). Human capital in growth regressions: how much difference does data quality make?. Journal of the European Economic Association 4(1), pp. 136

Gennaioli, N., La Porta, R, Lopezde-Silanes, F. et Shleifer, A. (2013). Human capital and Regional Development. The Quarterly Journal of Economics (2013), pp.105-164.

Guellouz, A., Masmoudi, A. et Smida, M. (2015). Histoire Générale de la Tunisie : Les Temps modernes.Sud Editions 2015.

Guiso, L., Sapienza, P. et Zingales L. (2008a), Social Capital as Good Culture. Journal of the European Economic Association 2008.

Guiso, L., Sapienza, P. et Zingales L.(2008b). Long Term Persistence. Journal of European Economic Association, December 2016, Vol. 14(6), pp.1401-1436.
Guiso, L., Sapienza, P. et Zingales L. (2010). Civic capital as the Missing Link. NBER WP Series 15845, NBER, 1050 Massachusetts Avenue, Cambridge, MA 02138, March 2010. Journal of Economics, v. 3 , pp.905-950.

Harris, J. R. et Todaro, M. (1970). Migration, unemployment and development: a two-sector analysis. The American economic review, Vol. 60(1), pp. 126-142 .

IMF (2015), Causes and Consequences of Inequality: a Global Perspective, prepared by Era Dabla-Norris, Kalpana Kochhar, Frantisek Ricka, Nujin Suphaphiphat, and Evridiki Tsounta; Strategy Policy and Review department, IMF, June 2015.

INS,

http://www.ins.tn/fr/statistiques

ITSEQ (2018), Indicateur de développement régional : méthodologie et résultats ; Institut Tunisien de la Compétitivité et des Etudes Quantitatives, note d'analyse No 1-2015, mai 2018.

Krueger A. et Lindhal M. (2001). Education for growth: why and for whom?, Journal of Economic Literature, 29, pp.1101-1136

Mankiw, G., Romer, D. et Weil, D. (1992). A Contribution to the Empirics of Economic Growth. Quarterly Journal of Economics, 107(2), pp.407-437.

Milanovic, B. (2016). Global Inequality: A New Approach for the Age of Globalization. Cambridge: Harvard University Press. 
MEHAT (Ministère de

l'Équipement, de l'Habitat et de l'Aménagement du Territoire)2 (2020). Atlas Numériques des

Gouvernorats ",

https://www.google.com/searc h? safe $=$ active $\alpha$ biw $=1093 \delta$ bih $=5$

20\&sxsrf=ALeKk03hbl8HlyHtJ2Ok id63nr64ukNK9w\%3A1589743428

108\&ei=RI_BXpWQBtXigweZipH4

Aw $\delta q=a t l a s+d u+g o u v e r n o r a t+d$ e+jendouba\&oq=gouvernorat+ de+jendouba\&gs_Icp=CgZwc3k tYWIQARgFMgIIADICCAAYAggA MgYIABAWEB4yBggAEBYQHjIGC AAQFhAeMgIlJjICCCY6BAgjECc6 BAgAEEM6BwgAEIMBEEM6BwgAE BQQhWI6CAgAEBYQChAeUMKIWI ViYLOIAWgACAB4AIABIAGIAYwck gEGMC4yMi4xmAEAoAEBqgEHZ3 dzLXdpeg\&sclient=psyab\#spf $=1589743830710$

OECD (2007). OECD Insights: Human Capital," https://www.oecd.org/insights/3 7966934.pdf

Piketty, T. (2001). Les inégalités dans le long terme. Inégalités Economiques, Rapport du Conseil d'Analyse Economique.
Piketty, T. (2013). Le capital au XXIè Siècle. Editions du Seuil, les livres du nouveau monde, Septembre 2013.

Porter, M. E. (1990). The competitive Advantage of Nations. The Free Press 1990.

Portes, A. (1998). Social Capital: Its Origins and Applications in Modern Sociology. Inheritability of Generalized Trust, Public Opinion Quarterly, v. 72 (4), pp.725-740.

Putnam, R.D. (1993). Making Democracy Work, Princeton: Princeton University Press.

Putnam, R.D. (1995a). Tuning In, Tuning Out: The Strange Disappearance of Social Capital in America. Political Science and Politics, vol. 28(4), pp.664-683.

Putnam, R.D. (1995b). The Case of Missing Social Capital. Harvard University Working

Putnam, R.D. (2000). Bowling Alone: The Collapse and Revival of American Community. New York: Simon and Schuster.

Ravallion, M. (2018). Inequality and Globalization: A Review Essay. Georgetown University, Journal of Economic Literature 2018, 56(2), pp.620-642

Schultz, T. (1961), Investment in Human capital. American Economic Review, Vol. 51 Nol, 1961.

Slim, H., Mahjoubi, A., Belkhoja, K. et Ennabli, A. (2006). Histoire générale de la Tunisie : L'Antiquité, 2006 Sud Editions, Tunis.

Tabellini, G.(2008). The Scope of Cooperation: Values and Incentive. The Quarterly Journal of Economics, 123(3), pp. 905950

Uslaner, E M. (2008). Where You Stand Depends Upon Where Your Grandparents Sat: The Inheritability of Generalized Trust, Department of Government and Politics. University of Maryland-College Park, College Park, MD. 20742 , 


\section{ANNEXES}

Tableau A 1. Régression Logit de l'entrepreneuriat

Source : calculs des auteurs à partir de l'enquête sur les inégalités en Tunisie, INS 2019

\begin{tabular}{|l|c|c|c|}
\hline Entrepreneuriat & Odds Ratio & & Std. Err. \\
\hline Niveau d'éducation & 1,041 & $* * *$ & 0,054 \\
\hline Niveau d'éducation du père & 0,918 & $* * *$ & 0,133 \\
\hline Capital humain hérité & 3,069 & $*$ & 1,76 \\
\hline Constante & 0,067 & $* * *$ & 0,018 \\
\hline
\end{tabular}

Tableau A 2. Répartition des grands-pères du chef de ménage par gouvernorat et niveau de compétence (nombre et \%)

Source : calculs des auteurs à partir de l'enquête sur les inégalités en Tunisie, INS 2019

\begin{tabular}{|l|c|c|c|c|c|}
\hline Compétence (Nombre \%) & Nabeul & Jendouba & Monastir & Kasserine & Total \\
\hline 0 & 0 & 1 & 2 & 0 & 3 \\
\hline & 0 & 33,33 & 66,67 & 0 & 100 \\
\hline 1 & 91 & 73 & 17 & 28 & 209 \\
\hline & 43,54 & 34,93 & 8,13 & 13,4 & 100 \\
\hline 2 & 321 & 317 & 333 & 347 & 1,318 \\
\hline 3 & 24,36 & 24,05 & 25,27 & 26,33 & 100 \\
\hline & 8 & 0 & 12 & 1 & 21 \\
\hline 4 & 38,1 & 0 & 57,14 & 4,76 & 100 \\
\hline 5 & 1 & 0 & 2 & 1 & 4 \\
\hline & 25 & 0 & 50 & 25 & 100 \\
\hline Total & 3 & 0 & 3 & 1 & 7 \\
\hline
\end{tabular}


Tableau A 3. Répartition des grands-pères du chef de ménage par gouvernorat et secteur d'activité

Source : calculs des auteurs à partir de l'enquête sur les inégalités en Tunisie, INS 2019

\begin{tabular}{|c|c|c|c|c|}
\hline \multirow[t]{2}{*}{ Secteur d'activité (nombre et \%) } & \multicolumn{4}{|c|}{ GOUVERNORAT } \\
\hline & NABEUL & JENDOUBA & MONASTIR & KASSERINE \\
\hline \multirow[t]{2}{*}{100} & 0 & 0 & 1 & 3 \\
\hline & 0,00 & 0,00 & 0,27 & 0,79 \\
\hline \multirow[t]{2}{*}{101} & 44 & 146 & 38 & 41 \\
\hline & 10,38 & 37,34 & 10,30 & 10,85 \\
\hline \multirow[t]{2}{*}{102} & 206 & 15 & 41 & 1 \\
\hline & 48,58 & 3,84 & 11,11 & 0,26 \\
\hline \multirow[t]{2}{*}{103} & 0 & 0 & 1 & 0 \\
\hline & 0 & 0,00 & 0,27 & 0 \\
\hline \multirow[t]{2}{*}{104} & 2 & 3 & 0 & 0 \\
\hline & 0,47 & 0,77 & 0 & 0 \\
\hline \multirow[t]{2}{*}{105} & 1 & 0 & 0 & 0 \\
\hline & 0,24 & 0,00 & 0 & 0 \\
\hline \multirow[t]{2}{*}{106} & 1 & 0 & 0 & 1 \\
\hline & 0,24 & 0,00 & 0 & 0,26 \\
\hline \multirow[t]{2}{*}{107} & 7 & 0 & 1 & 0 \\
\hline & 1,65 & 0,00 & 0,27 & 0 \\
\hline \multirow[t]{2}{*}{108} & 14 & 2 & 4 & 31 \\
\hline & 3,3 & 0,51 & 1,08 & 8,2 \\
\hline \multirow[t]{2}{*}{109} & 25 & 2 & 88 & 30 \\
\hline & 5,9 & 0,51 & 23,85 & 7,94 \\
\hline \multirow[t]{2}{*}{110} & 2 & 0 & 1 & 12 \\
\hline & 0,47 & 0,00 & 0,27 & 3,17 \\
\hline \multirow[t]{2}{*}{111} & 24 & 112 & 23 & 206 \\
\hline & 5,66 & 28,64 & 6,23 & 54,5 \\
\hline \multirow[t]{2}{*}{112} & 1 & 61 & 1 & 23 \\
\hline & 0,24 & 15,60 & 0,27 & 6,08 \\
\hline \multirow[t]{2}{*}{200} & 1 & 0 & 2 & 0 \\
\hline & 0,24 & 0,00 & 0,54 & 0 \\
\hline \multirow[t]{2}{*}{300} & 0 & 0 & 1 & 0 \\
\hline & 0 & 0,00 & 0,27 & 0 \\
\hline \multirow[t]{2}{*}{301} & 3 & 0 & 0 & 0 \\
\hline & 0,71 & 0,00 & 0 & 0 \\
\hline \multirow[t]{2}{*}{302} & 16 & 1 & 55 & 0 \\
\hline & 3,77 & 0,26 & 14,91 & 0 \\
\hline \multirow[t]{2}{*}{303} & 4 & 0 & 10 & 0 \\
\hline & 0,94 & 0,00 & 2,71 & 0 \\
\hline \multirow[t]{2}{*}{304} & 1 & 0 & 1 & 0 \\
\hline & 0,24 & 0,00 & 0,27 & 0 \\
\hline \multirow[t]{2}{*}{305} & 1 & 0 & 1 & 0 \\
\hline & 0,24 & 0,00 & 0,27 & 0 \\
\hline 308 & 2 & 0 & 1 & 0 \\
\hline
\end{tabular}




\begin{tabular}{|c|c|c|c|c|}
\hline & 0,47 & 0,00 & 0,27 & 0 \\
\hline \multirow[t]{2}{*}{309} & 8 & 0 & 1 & 0 \\
\hline & 1,89 & 0,00 & 0,27 & 0 \\
\hline \multirow[t]{2}{*}{310} & 0 & 0 & 2 & 2 \\
\hline & 0 & 0,00 & 0,54 & 0,53 \\
\hline \multirow[t]{2}{*}{312} & 0 & 0 & 3 & 1 \\
\hline & 0 & 0,00 & 0,81 & 0,26 \\
\hline \multirow[t]{2}{*}{400} & 32 & 29 & 54 & 14 \\
\hline & 7,55 & 7,42 & 14,63 & 3,7 \\
\hline \multirow[t]{2}{*}{500} & 14 & 15 & 30 & 3 \\
\hline & 3,3 & 3,84 & 8,13 & 0,79 \\
\hline \multirow[t]{2}{*}{600} & 0 & 0 & 2 & 1 \\
\hline & 0 & 0,00 & 0,54 & 0,26 \\
\hline \multirow[t]{2}{*}{700} & 1 & 0 & 0 & 0 \\
\hline & 0,24 & 0,00 & 0 & 0 \\
\hline \multirow[t]{2}{*}{800} & 0 & 0 & 0 & 1 \\
\hline & 0 & 0,00 & 0 & 0,26 \\
\hline \multirow[t]{2}{*}{900} & 2 & 1 & 0 & 1 \\
\hline & 0,47 & 0,26 & 0 & 0,26 \\
\hline \multirow[t]{2}{*}{1000} & 1 & 0 & 1 & 0 \\
\hline & 0,24 & 0,00 & 0,27 & 0 \\
\hline \multirow[t]{2}{*}{1100} & 11 & 4 & 6 & 7 \\
\hline & 2,59 & 1,02 & 1,36 & 1,85 \\
\hline \multirow[t]{2}{*}{ Total } & 424 & 391 & 369 & 378 \\
\hline & 100 & 100 & 100 & 100 \\
\hline
\end{tabular}


Tableau A 4. Indicateurs élémentaires composant l'indice de bien-être (IBE)-

Source : élaboration des auteurs à partir de l'enquête sur les inégalités en Tunisie, INS 2019

\begin{tabular}{|c|c|}
\hline Variable & Catégorie \\
\hline \multicolumn{2}{|l|}{ Monétaire } \\
\hline Les revenus du ménage & $\begin{array}{l}\text { Il s'agit de la somme de toutes les sources de revenus (du travail, } \\
\text { de la propriété, les pensions et les transferts publics et privés) des } \\
\text { deux conjoints }\end{array}$ \\
\hline \multicolumn{2}{|l|}{ Non monétaires } \\
\hline \multirow{10}{*}{ Type de logement } & Maison traditionnelle (Dar arbi) \\
\hline & Logement jumelé \\
\hline & Étage jumelé \\
\hline & Villa \\
\hline & Étage villa supérieur \\
\hline & RDC villa \\
\hline & Appartement \\
\hline & Studio \\
\hline & Houche, borj, (logement très modeste) \\
\hline & Taudis (gourbi, maamra, kib). \\
\hline Nombre de pièces & De là 9 \\
\hline \multirow{3}{*}{ Mode de propriété } & Propriétaire \\
\hline & Locataire \\
\hline & Gratuit \\
\hline \multirow{2}{*}{ Mode d'éclairage } & STEG $^{12}$ avec facture \\
\hline & STEG sans facture \\
\hline \multirow{2}{*}{ Gaz naturel } & Oui \\
\hline & Non \\
\hline \multirow{5}{*}{ Eau } & SONEDE ${ }^{13}$ avec facture \\
\hline & SONEDE sans facture \\
\hline & Puits privé \\
\hline & Fontaine \\
\hline & Source non contrôlée \\
\hline La distance séparant le domicile est : & De $0 \mathrm{~km}$ à $4 \mathrm{~km}$ ou plus \\
\hline \multicolumn{2}{|l|}{ École } \\
\hline \multicolumn{2}{|l|}{ Collège } \\
\hline \multicolumn{2}{|l|}{ Lycée } \\
\hline \multicolumn{2}{|l|}{ Centre de santé } \\
\hline \multicolumn{2}{|l|}{ Hôpital local } \\
\hline \multicolumn{2}{|l|}{ Crèche ou Jardin d'enfants } \\
\hline \multicolumn{2}{|l|}{ Établissement de jeunes } \\
\hline \multicolumn{2}{|l|}{ Établissement sportif } \\
\hline Centre commercial ou de service & \\
\hline
\end{tabular}

${ }_{12}^{2}$ STEG : principal producteur et fournisseur national d'électricité et de gaz.
${ }^{13}$ SONEDE : producteur et distributeur national d'eau potable. 



\section{Qu'est-ce que le groupe AFD ?}

Le groupe Agence française de développement (AFD) est un établissement public qui finance, accompagne et accélère les transitions vers un monde plus juste et durable. Plateforme française d'aide publique au développement et d'investissement de développement durable, nous construisons avec nos partenaires des solutions partagées, avec et pour les populations du Sud.

Nos équipes sont engagées dans plus de 4000 projets sur le terrain, dans les Outre-mer et dans 115 pays, pour les biens communs de l'humanité - le climat, la biodiversité, la paix, l'égalité femmes-hommes, l'éducation ou encore la santé. Nous contribuons ainsi à l'engagement de la développement durable.

Pour un monde en commun.

Directeur de publication Rémy Rioux Directeur de la rédaction Thomas Mélonio

Dépôt légal $3^{\mathrm{e}}$ trimestre 2020

ISSN 2492 - 2846 @ AFD

Création graphique MeMo, Juliegilles, D. Cazeils

Conception et réalisation AFD

Imprimé par le service reprographie de l'AFD

Pour consulter les autres publications

de la collection Papiers de recherche:

https://www.afd.fr/fr/collection/papiers-

de-recherche 